\title{
Global Stability Analysis of a Nonautonomous Stage-Structured Competitive System with Toxic Effect and Double Maturation Delays
}

\author{
Chao Liu ${ }^{1,2}$ and Yuanke $\mathrm{Li}^{1,3}$ \\ ${ }^{1}$ Institute of Systems Science, Northeastern University, Shenyang 110004, China \\ ${ }^{2}$ State Key Laboratory of Integrated Automation of Process Industry, Northeastern University, Shenyang 110004, China \\ ${ }^{3}$ Institute of Information and Computational Science, Northeastern University at Qinhuangdao, Qinhuangdao 066004, China
}

Correspondence should be addressed to Chao Liu; singularsystem@163.com

Received 25 February 2014; Revised 19 August 2014; Accepted 22 August 2014; Published 19 October 2014

Academic Editor: Zhiming Guo

Copyright (C) 2014 C. Liu and Y. Li. This is an open access article distributed under the Creative Commons Attribution License, which permits unrestricted use, distribution, and reproduction in any medium, provided the original work is properly cited.

\begin{abstract}
We investigate a nonautonomous two-species competitive system with stage structure and double time delays due to maturation for two species, where toxic effect of toxin liberating species on nontoxic species is considered and the inhibiting effect is zero in absence of either species. Positivity and boundedness of solutions are analytically studied. By utilizing some comparison arguments, an iterative technique is proposed to discuss permanence of the species within competitive system. Furthermore, existence of positive periodic solutions is investigated based on continuation theorem of coincidence degree theory. By constructing an appropriate Lyapunov functional, sufficient conditions for global stability of the unique positive periodic solution are analyzed. Numerical simulations are carried out to show consistency with theoretical analysis.
\end{abstract}

\section{Introduction}

In recent years, many research efforts have been made on competitive Lotka-Volterra system with stage structure and time delay. By incorporating a constant time delay into single species model, a stage-structured model is proposed in the pioneering work [1], where time delay reflects a delayed birth of immature population and a reduced survival of immature population to their maturity. The model system takes the following form:

$$
\begin{gathered}
\dot{x}_{i}(t)=\alpha x_{m}(t)-\gamma x_{i}(t)-\alpha e^{-\gamma \tau} x_{m}(t-\tau), \\
\dot{x}_{m}(t)=\alpha e^{-\gamma \tau} x_{m}(t-\tau)-\beta x_{m}^{2}(t),
\end{gathered}
$$

where $x_{i}(t)$ and $x_{m}(t)$ represent the immature population and mature population density at time $t$, respectively. $\alpha>$ 0 denotes the birth rate of immature population; $\gamma>0$ stands for the death rate of immature population. $\beta>0$ is the death and overcrowding rate of mature population. $\tau$ denotes time of immature population to maturity. The term $\alpha e^{-\gamma \tau} x_{m}(t-\tau)$ represents the immature species which are born at time $t-\tau$ and survive at time $t$ with immature death rate $\gamma$ and therefore represents transformation of immature species to mature species. It is found that all ecologically relevant solutions tend to the positive equilibrium solution as time $t \rightarrow \infty$, and various aspects of the above proposed system including positivity and boundedness of solutions are discussed in [1].

Zeng et al. propose a nonautonomous competitive twospecies model with stage structure in one species in [2], where conditions of permanence are obtained. Furthermore, existence and asymptotic stability of periodic solution are proved under some assumptions if the proposed model turns out to be a periodic system. A two-species Lotka-Volterra type competition model with stage structure for both species is proposed and investigated in [3], where the individuals of each species are classified as immature and mature. By constructing a suitable Lyapunov function, sufficient conditions 
are derived for the global stability of nonnegative equilibria of the proposed model in the case of constant coefficients. Furthermore, a set of easily verifiable sufficient conditions are obtained for the existence of positive periodic solution when coefficients are assumed to be positively continuous periodic functions. In [4], there is a time delayed periodic system which describes the competition among mature populations. The evolutionary behavior of model system is analyzed and some sufficient conditions for competitive coexistence and exclusion are obtained.

A nonautonomous competitive Lotka-Volterra system is studied in [5]; it reveals a computable necessary and sufficient condition for the system to be totally permanent when the growth rates have averages and the interaction coefficients are nonnegative constants. Along with this research, permanence for a class of competitive Lotka-Volterra systems is discussed in [6] which extends the work done in [5], and a computable necessary and sufficient condition is found for the permanence of all subsystems of the system and its small perturbation on the interaction matrix. In [7], a twospecies competitive model with stage structure is discussed, and the dynamics of coupled system of semilinear parabolic equations with time delays are investigated, which show that the introduction of diffusion does not affect the permanence and extinction of the species, though the introduction of stage structure brings negative effect on it. In [8], sufficient conditions are obtained for the existence of a unique, globally attractive, strictly positive (componentwise), almost periodic solution of a nonautonomous, almost periodic competitive two-species model with a stage structure in one species. An example together with its numeric simulations shows the feasibility of our main results, which generalize the main results of Zeng et al. [2]. According to two types of wellknown periodic single species population growth models with time delay, two corresponding periodic competitive systems with multiple delays are proposed in [9], and the same criteria for the existence and globally asymptotic stability of positive periodic solutions of the above two competitive systems are derived. In [10], a discrete periodic competitive model with stage structure is established, and some sufficient and realistic conditions are obtained for existence of a positive periodic solution of the proposed system. In [11], a periodic nonautonomous competitive stage-structured system with infinite delay is considered, where the adult members of $n$-species are in competition. For each of the $n$-species the model incorporates a time delay which represents the time from birth to maturity of that species. Infinite delay is introduced which denotes the influential effect of the entire past history of the system on the current competition interactions. By using the comparison principle, if the growth rates are sufficiently large, then the solutions are uniformly permanent. Then, by using Horn's fixed point theorem, the existence of positive periodic solution of the system with finite delay is discussed. Finally, it is proved that even the system with infinite delay admits a positive periodic solution.

In [12], a nonautonomous predator-prey system with discrete time delay is studied, where there is epidemic disease in the predator. By using some techniques of the differential inequalities and delay differential inequalities, the permanence of system is discussed under some appropriate conditions. When all the coefficients of the system are periodic, the existence and global attractivity of the positive periodic solution are studied by Mawhin's continuation theorem and constructing a suitable Lyapunov functional. Furthermore, when the coefficients of the system are not absolutely periodic but almost periodic, sufficient conditions are also derived for the existence and asymptotic stability of the almost periodic solution. In [13], general $n$ species nonautonomous Lotka-Volterra competitive systems with pure-delays and feedback controls are discussed. New sufficient conditions, for which a part of the $n$-species remains permanent, are established by applying the method of multiple Lyapunov functionals and introducing a new analysis technique.

By utilizing Brouwer fixed point theorem and constructing a suitable Lyapunov function, the periodic solution and global stability for a nonautonomous competitive LotkaVolterra diffusion system are investigated in [14]; it can be found that the system has a unique periodic solution which is globally stable under some appropriate conditions. In [15], a delay differential equation model for the interaction between two species is investigated. The maturation delay for each species is modelled as a distribution, to allow for the possibility that individuals may take different amount of time to maturity. Positivity and boundedness of the solutions are studied, and global stability is analyzed for each equilibrium. A Lotka-Volterra competitive system with infinite delay and feedback controls is proposed in [16]. By using Mawhin's continuation theorem of coincidence degree theory, an impulsive nonautonomous Lotka-Volterra predator-prey system with harvesting terms is investigated in [17]. Some sufficient conditions for the existence of multiple positive almost periodic solutions for the system under consideration are discussed. Furthermore, existence of multiple positive almost periodic solutions to other types of population systems can be studied by using the same method obtained in this paper. By using the method of multiple Lyapunov functionals and by developing a new analysis technique, some sufficient conditions are obtained that guarantee that some of the $n$ species are driven to extinction. A three-dimensional nonautonomous competitive Lotka-Volterra system is considered in [18]; it is shown that if the growth rates are positive, bounded, and continuous functions, and the averages of the growth rates satisfy certain inequalities, then any positive solution has the property that one of its components vanishes. In [19], an almost periodic multispecies Lotka-Volterra mutualism system with time delays and impulsive effects is investigated. By using the theory of comparison theorem and constructing a suitable Lyapunov functional, sufficient conditions which guarantee the existence and uniqueness and global asymptotical stability of almost periodic solution of this system are obtained.

It is well known that the effect of toxins on ecological systems is an important issue from mathematical and experimental points of view $[20,21]$. The first mathematical model to represent the toxic liberating interaction between two competing species is introduced by Maynard Smith [22]. The model is based upon a two-species Lotka-Volterra 
competition model with an additional term to take into account the effect of toxic substances released by one species to another, which takes the following form:

$$
\begin{aligned}
& \dot{N}_{1}(t)=N_{1}(t)\left[\alpha_{1}-\beta_{1} N_{1}(t)-c_{1} N_{2}(t)-\rho_{1} N_{1}(t) N_{2}(t)\right], \\
& \dot{N}_{2}(t)=N_{2}(t)\left[\alpha_{2}-\beta_{2} N_{2}(t)-c_{2} N_{1}(t)-\rho_{2} N_{1}(t) N_{2}(t)\right],
\end{aligned}
$$

where $N_{1}(t)$ and $N_{2}(t)$ represent the density of two competing species at time $t$, respectively. $\alpha_{1}$ and $\alpha_{2}$ denote the birth rate of $N_{1}(t)$ species and $N_{2}(t)$ species, respectively. $\beta_{1}$ and $\beta_{2}$ are the rate of intraspecific competition term for the first and second species, respectively. $c_{1}$ and $c_{2}$ stand for the rate of interspecific competition, respectively. $\rho_{1}$ and $\rho_{2}$ represent the toxic inhibition rate for the first species by the second species and vice versa. By considering that $\rho$ denotes the rate of toxic inhibition for the nontoxic species $N_{1}(t)$ released by the toxin liberating species $N_{2}(t)$ and all other parameters share the same biological interpretations mentioned in model system (2), work done in [22] is extended in [23] and the generalized model system is as follows:

$$
\begin{aligned}
\dot{N}_{1}(t)= & N_{1}(t)\left[\alpha_{1}-\beta_{1} N_{1}(t)-c_{1} N_{2}(t)-\rho N_{1}(t) N_{2}^{2}(t)\right], \\
& \dot{N}_{2}(t)=N_{2}(t)\left[\alpha_{2}-\beta_{2} N_{2}(t)-c_{2} N_{1}(t)\right]
\end{aligned}
$$

where the toxic substance producing action follows the mathematical term $\rho N_{1}^{2}(t) N_{2}^{2}(t)$ [23].

It should be noted that models of the persistence and extinction of a population or community in a polluted environment have been investigated in [23]. But all of those papers have a basic assumption that the capacity of the environment is so large that the change of toxicant in the environment that comes from uptake and egestation by the organisms can be neglected. This assumption is not made in $[24,25]$, some sufficient conditions on persistence or extinction of a population have been obtained, and the threshold between the two has also been obtained for most situations. In [26, 27], there are modified delay differential equation models of the growth of two species of plankton having competitive and allelopathic effects on each other. By using the continuation theorem of coincidence degree theory, a set of easily verifiable sufficient conditions are obtained for the existence of positive periodic solutions for this model. Recently, some discussions and investigations of the nonautonomous competitive model with toxic effects are made. A periodic competitive stage-structured Lotka-Volterra model with the effects of toxic substances is investigated in [28]. It is shown that toxic substances play an important role in the extinction of species. A set of sufficient conditions guarantee that one of the components is driven to extinction while the other is globally attractive. The dynamical behavior of a two-species competitive system affected by toxic substances is investigated in [21], where each species produces a substance toxic to the other species. Boundedness and local and global stabilities are also addressed. It should be noted that toxic interaction follows the mathematical term suggested in model system (2) and each mature individual produces a substance toxic to the other mature individuals only when the other mature individual is present, and the immature individual is not affected by the toxicant [21]. However, to the author's best knowledge, dynamical behavior and stability analysis of nonautonomous stage-structured competitive system with toxin liberating species and nontoxic species have not been investigated. Generally speaking, it takes some time for a species to reach maturity to produce the toxicant; then toxin liberating mature individual produces a substance toxic to the nontoxic mature individuals only. The inhibiting effect is zero in absence of either species, and the immature individual of each species is not affected by the toxicant. Furthermore, the species compete each other for the limited life resource within closed environment, but this competition only happens among the mature individuals and does not involve the immature individuals. Consequently, it is necessary to investigate the dynamic effect of stage structure and toxic effect on the population dynamics of two-species competitive system with toxin liberating species and nontoxic species.

The rest section of this paper is organized as follows: a nonautonomous two-species competitive model is established in the second section. Stage structure and maturation delay for each species are introduced, and toxic effect of toxin liberating species on nontoxic species is considered. In the third section, qualitative analyses are performed to investigate the effect of stage structure and toxic substances on the dynamical behavior of two-species competitive model system. The positivity and boundedness of solutions are analytically studied. By utilizing some comparison arguments, an iterative technique is proposed to discuss permanence of the species within competitive system. Furthermore, existence of positive periodic solutions is considered based on continuation theorem of coincidence degree theory. By constructing an appropriate Lyapunov functional, sufficient conditions for global stability of the unique positive periodic solution are analyzed. Numerical simulations are provided to support the theoretical findings obtained in this paper. Finally, this paper ends with a conclusion.

\section{Model Formulation}

In this paper, the effect of stage structure and toxic substances on the dynamical behavior of two-species competitive model system is investigated under the following five hypotheses, which are given as follows.

(H1) Two competing species, that is, nontoxic species and toxin liberating species, are considered in this paper. It is assumed that each species is divided into two-stage groups, and the immature and mature individuals are divided by a fixed period. $x_{1}(t)$ and $y_{1}(t)$ represent immature population density of nontoxic species and toxin liberating species at time $t$, respectively; $x_{2}(t)$ and $y_{2}(t)$ denote mature population density of nontoxic species and toxin liberating species at time $t$, respectively. 
(H2) $\omega$-periodic continuous functions $\alpha_{1}(t)>0$ and $\alpha_{2}(t)>0$ denote the birth rate of immature population of nontoxic species and toxin liberating species at time $t$, respectively. $\omega$-periodic continuous functions $\gamma_{1}(t)>0$ and $\gamma_{2}(t)>0$ stand for the death rate of immature population of nontoxic species and toxin liberating species at time $t$, respectively. $\omega$-periodic continuous functions $\beta_{1}(t)>0$ and $\beta_{2}(t)>0$ are the death and overcrowding rate of mature population of nontoxic species and toxin liberating species at time $t$, respectively.

(H3) $\tau_{1}$ denotes time of immature nontoxic species to maturity. The term $\alpha_{1}\left(t-\tau_{1}\right) e^{-\int_{t-\tau_{1}}^{t} \gamma_{1}(s) \mathrm{d} s} x_{2}\left(t-\tau_{1}\right)$ represents the immature nontoxic species which are born at time $t-\tau_{1}$ and survive at time $t$ with immature death rate. $\tau_{2}$ denotes time of immature toxin liberating species to maturity. The term $\alpha_{2}(t-$ $\left.\tau_{2}\right) e^{-\int_{t-\tau_{2}}^{t} \gamma_{2}(s) \mathrm{d} s} y_{2}\left(t-\tau_{2}\right)$ represents the immature toxin liberating species which are born at time $t-\tau_{2}$ and survive at time $t$ with immature death rate.

(H4) For toxin liberating species, it takes some time to attain its level of maturity to produce the toxic substances, and toxin liberating mature individual produces a substance toxic to the nontoxic mature individuals only. The inhibiting effect is zero in absence of either species, and the immature individual of each species is not affected by the toxicant. Based on model system (3), the toxic effect released by toxin liberating species on nontoxic species is described by the mathematical term $\rho(t) x_{2}^{2}(t) y_{2}^{2}(t)$, where toxic inhibition rate is represented by an $\omega$ periodic continuous function $\rho(t)>0$.

(H5) Nontoxic species and toxin liberating species compete each other for the common resource within closed environment, but this competition only happens among the mature individuals and does not involve the immature individuals. $\omega$-periodic continuous function $c_{1}(t)>0$ represents interspecific competition rate for the mature nontoxic species by the mature toxin liberating species, and $\omega$-periodic continuous function $c_{2}(t)>0$ represents interspecific competition rate for the mature toxin liberating species by the mature nontoxic species.

Based on hypotheses (H1)-(H5), a nonautonomous stagestructured competitive model with toxic effect and double maturation delays is established as follows:

$$
\begin{aligned}
\dot{x}_{1}(t)= & \alpha_{1}(t) x_{2}(t)-\gamma_{1}(t) x_{1}(t) \\
& -\alpha_{1}\left(t-\tau_{1}\right) e^{-\int_{t-\tau_{1}}^{t} \gamma_{1}(s) \mathrm{d} s} x_{2}\left(t-\tau_{1}\right), \\
\dot{x}_{2}(t)= & \alpha_{1}\left(t-\tau_{1}\right) e^{-\int_{t-\tau_{1}}^{t} \gamma_{1}(s) \mathrm{d} s} x_{2}\left(t-\tau_{1}\right)-\beta_{1}(t) x_{2}^{2}(t) \\
& -c_{1}(t) x_{2}(t) y_{2}(t)-\rho(t) x_{2}^{2}(t) y_{2}^{2}(t),
\end{aligned}
$$

$$
\begin{aligned}
\dot{y}_{1}(t)= & \alpha_{2}(t) y_{2}(t)-\gamma_{2}(t) y_{1}(t) \\
& -\alpha_{2}\left(t-\tau_{2}\right) e^{-\int_{t-\tau_{2}}^{t} \gamma_{2}(s) \mathrm{d} s} y_{2}\left(t-\tau_{2}\right), \\
\dot{y}_{2}(t)= & \alpha_{2}\left(t-\tau_{2}\right) e^{-\int_{t-\tau_{2}}^{t} \gamma_{2}(s) \mathrm{d} s} y_{2}\left(t-\tau_{2}\right)-\beta_{2}(t) y_{2}^{2}(t) \\
& -c_{2}(t) x_{2}(t) y_{2}(t) .
\end{aligned}
$$

In this paper, model system (4) is investigated with the following initial conditions:

$$
\begin{aligned}
& x_{i}(\theta)=\phi_{i}(\theta)>0, \quad-\tau_{1} \leq \theta \leq 0, \quad i=1,2, \\
& y_{i}(\theta)=\psi_{i}(\theta)>0, \quad-\tau_{2} \leq \theta \leq 0, i=1,2 .
\end{aligned}
$$

For the continuity of the initial conditions, it is required that

$$
\begin{aligned}
& x_{1}(0)=\int_{-\tau_{1}}^{0} \alpha_{1}(\theta) \phi_{2}(\theta) e^{\int_{0}^{\theta} \gamma_{1}(s) \mathrm{d} s} \mathrm{~d} \theta \\
& y_{1}(0)=\int_{-\tau_{2}}^{0} \alpha_{2}(\theta) \psi_{2}(\theta) e^{\int_{0}^{\theta} \gamma_{2}(s) \mathrm{d} s} \mathrm{~d} \theta
\end{aligned}
$$

\section{Qualitative Analysis of Model System}

In this section, qualitative analysis of the nonautonomous model system (4) is performed, which is utilized to discuss dynamic effect of toxic effect and maturation delay on population dynamics. The positivity and boundedness of solutions are analytically studied. By utilizing some comparison arguments, an iterative technique is proposed to discuss permanence of the species within competitive system. Furthermore, existence of positive periodic solutions is investigated based on continuation theorem of coincidence degree theory. By constructing an appropriate Lyapunov functional, sufficient conditions for global stability of the unique positive periodic solution are analyzed.

Some mathematical notations are adopted for convenience of the following statement:

$$
f^{L}=\min _{t \in[0, \omega]}|f(t)|, \quad f^{M}=\max _{t \in[0, \omega]}|f(t)|,
$$

where $f(t)$ is a $\omega$-periodic continuous function.

\subsection{Permanence of Solutions}

Theorem 1. Solutions of model system (4) with initial conditions (5) and (6) are positive for all $t>0$.

Proof. Firstly, we show that $x_{2}(t)>0$ for all $t>0$. Otherwise, if it is false, since $x_{2}(t)>0$ for all $t \in\left[-\tau_{1}, 0\right]$, then it can be derived that there exists a $t_{1}>0$ such that $x_{2}\left(t_{1}\right)=0$.

Define $t_{0}=\inf \left\{t>0 \mid x_{2}(t)=0\right\}$. According to the definition of $t_{0}$, it can be obtained that

$$
\dot{x}_{2}\left(t_{0}\right) \leq 0 \text {. }
$$



that

It follows from the second equation of model system (4)

$$
\begin{aligned}
& \dot{x}_{2}\left(t_{0}\right) \\
& = \begin{cases}\alpha_{1}\left(t_{0}-\tau_{1}\right) e^{-\int_{t_{0}-\tau_{1}}^{t_{0}} \gamma_{1}(s) \mathrm{d} s} \phi_{2}\left(t_{0}-\tau_{1}\right)>0, & 0 \leq t_{0} \leq \tau_{1}, \\
\alpha_{1}\left(t_{0}-\tau_{1}\right) e^{-\int_{t_{0}-\tau_{1}}^{t_{1}} \gamma_{1}(s) \mathrm{d} s} x_{2}\left(t_{0}-\tau_{1}\right)>0, & t>\tau_{1},\end{cases}
\end{aligned}
$$

and it is easy to show that $\dot{x}_{2}\left(t_{0}\right)>0$, which is a contradiction to (8). Hence, $x_{2}(t)>0$ for all $t>0$.

By a direct computation, it follows from the first equation of model system (4) that

$$
x_{1}(t)=\int_{t-\tau_{1}}^{t} \alpha_{1}(s) e^{\int_{t}^{s} \gamma_{1}(m) \mathrm{d} s} x_{2}(s) \mathrm{d} s,
$$

since $x_{2}(t)>0$ for all $t>0$; it is easy to show that $x_{1}(t)>0$ for all $t>0$ based on (10).

By utilizing the similar proof, it can be obtained that $y_{1}(t)>0$ and $y_{2}(t)>0$ for all $t>0$. Consequently, solutions of model system (4) with initial conditions (5) and (6) are positive for all $t>0$.

Theorem 2. Solutions of model system (4) with initial conditions (5) and (6) are ultimately bounded.

Proof. Let $w(t)=x_{1}(t)+x_{2}(t)+y_{1}(t)+y_{2}(t)$, where $\left(x_{1}(t), x_{2}(t), y_{1}(t), y_{2}(t)\right)$ is an arbitrary positive solution of model system (4) with the initial conditions (5) and (6).

Calculating the derivative of $w(t)$ along the solution of model system (4) gives that

$$
\begin{aligned}
\dot{w}(t) \leq & \left(\alpha_{1}(t)+\gamma_{1}(t)\right) x_{2}(t)-\gamma_{1}(t)\left(x_{1}(t)+x_{2}(t)\right) \\
& -\beta_{1}(t) x_{2}^{2}(t) \\
& +\left(\alpha_{2}(t)+\gamma_{2}(t)\right) y_{2}(t)-\gamma_{2}(t)\left(y_{1}(t)+y_{2}(t)\right) \\
& -\beta_{2}(t) y_{2}^{2}(t) \\
\leq & \left(\alpha_{1}^{M}+\gamma_{1}^{M}\right) x_{2}(t)-\beta_{1}^{L} x_{2}^{2}(t)-r_{1}^{L}\left(x_{1}(t)+x_{2}(t)\right) \\
& +\left(\alpha_{2}^{M}+\gamma_{2}^{M}\right) y_{2}(t)-\beta_{2}^{L} y_{2}^{2}(t)-r_{2}^{L}\left(y_{1}(t)+y_{2}(t)\right) \\
\leq & -\gamma^{L} w(t)+\left(\alpha_{1}^{M}+\gamma_{1}^{M}\right) x_{2}(t)-\beta_{1}^{L} x_{2}^{2}(t) \\
& +\left(\alpha_{2}^{M}+\gamma_{2}^{M}\right) y_{2}(t)-\beta_{2}^{L} y_{2}^{2}(t) \\
\leq & -\gamma^{L} w(t)+\frac{\left(\alpha_{1}^{M}+\gamma_{1}^{M}\right)^{2}}{4 \beta_{1}^{L}}+\frac{\left(\alpha_{2}^{M}+\gamma_{2}^{M}\right)^{2}}{4 \beta_{2}^{L}},
\end{aligned}
$$

where $\gamma^{L}=\min \left\{\gamma_{1}^{L}, \gamma_{2}^{L}\right\}$

By using the standard comparison principle [20], it follows from (11) that

$$
w(t) \leq \frac{\beta_{2}^{L}\left(\alpha_{1}^{M}+\gamma_{1}^{M}\right)^{2}+\beta_{1}^{L}\left(\alpha_{2}^{M}+\gamma_{2}^{M}\right)^{2}}{4 \gamma^{L} \beta_{1}^{L} \beta_{2}^{L}},
$$

which implies that any solution of model system (4) with initial conditions (5) and (6) is ultimately bounded.

Lemma 3 (see [29]). Consider the following differential equation:

$$
\dot{x}(t)=a x(t-\tau)-b x(t)-c x^{2}(t),
$$

where $a, b, c, \tau>0$ and $x(t)>0$ for $-\tau \leq t \leq 0$; we have that

(i) if $a>b$, then $\lim _{t \rightarrow+\infty} x(t)=(a-b) / c$;

(ii) if $a<b$, then $\lim _{t \rightarrow+\infty} x(t)=0$.

Lemma 4 (see [29]). Consider the following differential equation:

$$
\dot{x}(t)=d x(t-\sigma)-e x^{2}(t),
$$

where $d, e, \sigma>0$ and $x(t)>0$ for $-\sigma \leq t \leq 0$; we have

$$
\lim _{t \rightarrow+\infty} x(t)=\frac{d}{e}
$$

Definition 5 (see [30]). Model system

$$
\dot{X}(t)=f\left(t, X_{t}(\theta)\right),
$$

where $t \geq 0, \theta \in[-\tau, 0], X \in \mathbb{R}^{n}$. Model system (16) is said to be permanent if, for any solution $X(t, \phi)$, there exists a constant $m>0$ and $T=T(\phi)$ such that $X(t)>m$ for all $t>T$.

Definition 6 (see [30]). The domain $D \in \mathbb{C}^{n}$ is said to be an ultimately bounded domain, if $D$ is a closed, bounded subset of $\mathbb{C}^{n}$, and there exists a constant $T=T(\phi)$ such that $X_{t}(\theta) \in$ $D$ for all $t>T$.

Theorem 7. If $\alpha_{1}^{L} \beta_{2}^{L}>c_{1}^{M} \alpha_{2}^{M}$ and $\alpha_{2}^{L} \beta_{1}^{L}>c_{2}^{M} \alpha_{1}^{M}$, then model system (4) is permanent with initial conditions (5) and (6).

Proof. According to the second equation of model system (4) and Theorem 1 , we get that

$$
\dot{x}_{2}(t) \leq \alpha_{1}^{M} e^{-\gamma_{1}^{L} \tau_{1}} x_{2}\left(t-\tau_{1}\right)-\beta_{1}^{L} x_{2}^{2}(t) .
$$

By virtue of Lemma 4 and (17), there exists a positive time $T_{1}$ such that, for sufficiently small $\epsilon>0$ and $t \geq T_{1}$, it yields

$$
x_{2}(t) \leq \frac{\alpha_{1}^{M} e^{-\gamma_{1}^{L} \tau_{1}}}{\beta_{1}^{L}}+\epsilon:=M_{2}^{(1)} .
$$

By rearranging (10), it can be obtained that

$$
x_{1}(t)=e^{-\int_{0}^{t} \gamma_{1}(s) \mathrm{d} s} \int_{t-\tau_{1}}^{t} \alpha_{1}(s) e^{\int_{0}^{s} \gamma_{1}(m) \mathrm{d} m} x_{2}(s) \mathrm{d} s .
$$

For any $t \geq T_{1}$, it follows from (18) and (19) that

$$
x_{1}(t) \leq \frac{a_{1}^{M} M_{2}^{(1)}\left(1-e^{-\gamma_{1}^{M} \tau_{1}}\right)}{\gamma_{1}^{L}}:=M_{1}^{(1)} .
$$


Based on the fourth equation of model system (4) and Theorem 1, it can be obtained that

$$
\dot{y}_{2}(t) \leq \alpha_{2}^{M} e^{-\gamma_{2}^{L} \tau_{2}} y_{2}\left(t-\tau_{2}\right)-\beta_{2}^{L} y_{2}^{2}(t)
$$

holds for $t \geq T_{1}$.

By virtue of Lemma 4 and (21), there exists $T_{2}>T_{1}$ such that, for sufficiently small $\epsilon>0$ and $t \geq T_{2}$, it yields

$$
y_{2}(t) \leq \frac{\alpha_{2}^{M} e^{-\gamma_{2}^{L} \tau_{2}}}{\beta_{2}^{L}}+\epsilon:=M_{4}^{(1)} .
$$

By direct computing, it follows from the third equation of model system (4) that

$$
y_{1}(t)=e^{-\int_{0}^{t} \gamma_{2}(s) \mathrm{d} s} \int_{t-\tau_{2}}^{t} \alpha_{2}(s) e^{\int_{0}^{s} \gamma_{2}(m) \mathrm{d} m} y_{2}(s) \mathrm{d} s .
$$

For any $t \geq T_{2}$, it follows from (22) and (23) that

$$
y_{1}(t) \leq \frac{\alpha_{2}^{M} M_{4}^{(1)}\left(1-e^{-\gamma_{2}^{M} \tau_{2}}\right)}{\gamma_{2}^{L}}:=M_{3}^{(1)} .
$$

Furthermore, it follows from the second equation of model system (4) that

$$
\begin{aligned}
\dot{x}_{2}(t) \geq & \alpha_{1}^{L} e^{-\gamma_{1}^{M} \tau_{1}} x_{2}\left(t-\tau_{1}\right) \\
& -\left(\beta_{1}^{M}+\rho_{1}^{M}\left(M_{4}^{(1)}\right)^{2}\right) x_{2}^{2}(t)-c_{1}^{M} M_{4}^{(1)} x_{2}(t) .
\end{aligned}
$$

Based on Lemma 3 and (25), there exists $T_{3}>T_{2}$ and for any $t \geq T_{3}$ and sufficiently small $\epsilon>0$,

$$
x_{2}(t) \geq \frac{\alpha_{1}^{L} e^{-\gamma_{1}^{M} \tau_{1}}-c_{1}^{M} M_{4}^{(1)}}{\beta_{1}^{M}+\rho_{1}^{M}\left(M_{4}^{(1)}\right)^{2}}-\epsilon:=m_{2}^{(1)}
$$

holds provided that

$$
\alpha_{1}^{L} e^{-\gamma_{1}^{M} \tau_{1}}>c_{1}^{M} M_{4}^{(1)}
$$

According to (19), for any $t \geq T_{3}$, we get that

$$
x_{1}(t) \geq \frac{\alpha_{1}^{L}\left(1-e^{-\gamma_{1}^{L} \tau_{1}}\right) m_{2}^{(1)}}{\gamma_{1}^{M}}:=m_{1}^{(1)} .
$$

For any $t \geq T_{3}$, it follows from the fourth equation of model system (4) that

$$
\dot{y}_{2}(t) \geq \alpha_{2}^{L} e^{-\gamma_{2}^{M} \tau_{2}} y_{2}\left(t-\tau_{2}\right)-\beta_{2}^{M} y_{2}^{2}(t)-c_{2}^{M} M_{2}^{(1)} y_{2}(t) .
$$

Based on Lemma 3 and (29), there exists $T_{4}>T_{3}$ and for any $t \geq T_{4}$ and sufficiently small $\epsilon>0$,

$$
y_{2}(t) \geq \frac{\alpha_{2}^{L} e^{-\gamma_{2}^{M} \tau_{2}}-c_{2}^{M} M_{2}^{(1)}}{\beta_{2}^{M}}-\epsilon:=m_{4}^{(1)}
$$

holds provided that

$$
\alpha_{2}^{L} e^{-\gamma_{2}^{M} \tau_{2}}>c_{2}^{M} M_{2}^{(1)} .
$$

According to (23) and (30), it can be obtained that

$$
y_{1}(t) \geq \frac{\alpha_{2}^{L} m_{4}^{(1)}\left(1-e^{-\gamma_{2}^{L} \tau_{2}}\right)}{\gamma_{2}^{M}}:=m_{3}^{(1)} .
$$

According to the second equation of model system (4), we get that

$$
\begin{aligned}
\dot{x}_{2}(t) \leq & \alpha_{1}^{M} e^{-\gamma_{1}^{L} \tau_{1}} x_{2}\left(t-\tau_{1}\right) \\
& -\left(\beta_{1}^{L}+\rho^{L}\left(m_{4}^{(1)}\right)^{2}\right) x_{2}^{2}(t)-c_{1}^{L} m_{4}^{(1)} x_{2}(t) .
\end{aligned}
$$

By virtue of Lemma 3 and (33), there exists $T_{5}>T_{4}$ such that, for sufficiently small $\epsilon>0$ and $t \geq T_{5}$, it yields

$$
x_{2}(t) \leq \frac{\alpha_{1}^{M} e^{-\gamma_{1}^{L} \tau_{1}}-c_{1}^{L} m_{4}^{(1)}}{\beta_{1}^{L}+\rho^{L}\left(m_{4}^{(1)}\right)^{2}}+\epsilon:=M_{2}^{(2)},
$$

which holds provided that

$$
\alpha_{1}^{M} e^{-\gamma_{1}^{L} \tau_{1}}>c_{1}^{L} m_{4}^{(1)}
$$

For any $t \geq T_{5}$, it follows from (19) and (34) that

$$
x_{1}(t) \leq \frac{\alpha_{1}^{M} M_{2}^{(2)}\left(1-e^{-\gamma_{1}^{M} \tau_{1}}\right)}{\gamma_{1}^{L}}:=M_{1}^{(2)} .
$$

Based on the fourth equation of model system (4), it can be obtained that

$$
\dot{y}_{2}(t) \leq \alpha_{2}^{M} e^{-\gamma_{2}^{L} \tau_{2}} y_{2}\left(t-\tau_{2}\right)-\beta_{2}^{L} y_{2}^{2}(t)-c_{2}^{L} m_{2}^{(1)} y_{2}(t)
$$

holds for $t \geq T_{5}$.

By virtue of Lemma 3 and (37), there exists $T_{6}>T_{5}$ such that, for sufficiently small $\epsilon>0$ and $t \geq T_{6}$, it yields

$$
y_{2}(t) \leq \frac{\alpha_{2}^{M} e^{-\gamma_{2}^{L} \tau_{2}}-c_{2}^{L} m_{2}^{(1)}}{\beta_{2}^{L}}+\epsilon:=M_{4}^{(2)},
$$

which holds provided that

$$
\alpha_{2}^{M} e^{-\gamma_{2}^{L} \tau_{2}}>c_{2}^{L} m_{2}^{(1)} .
$$

For any $t \geq T_{6}$, it follows from (23) and (38) that

$$
y_{1}(t) \leq \frac{\alpha_{2}^{M} M_{4}^{(2)}\left(1-e^{-\gamma_{2}^{M} \tau_{2}}\right)}{\gamma_{2}^{L}}:=M_{3}^{(2)} .
$$

Furthermore, it follows from the second equation of model system (4) that

$$
\begin{aligned}
\dot{x}_{2}(t) \geq & \alpha_{1}^{L} e^{-\gamma_{1}^{M} \tau_{2}} x_{2}\left(t-\tau_{1}\right) \\
& -\left(\beta_{1}^{M}+\rho^{M}\left(M_{4}^{(2)}\right)^{2}\right) x_{2}^{2}(t)-c_{1}^{M} M_{4}^{(2)} x_{2}(t) .
\end{aligned}
$$


Based on Lemma 3 and (41), there exists $T_{7}>T_{6}$ and, for any $t \geq T_{7}$ and sufficiently small $\epsilon>0$,

$$
x_{2}(t) \geq \frac{\alpha_{1}^{L} e^{-\gamma_{1}^{M} \tau_{1}}-c_{1}^{M} M_{4}^{(2)}}{\beta_{1}^{M}+\rho^{M}\left(M_{4}^{(2)}\right)^{2}}-\epsilon:=m_{2}^{(2)}
$$

holds provided that

$$
\alpha_{1}^{L} e^{-\gamma_{1}^{M} \tau_{1}}>c_{1}^{M} M_{4}^{(2)} .
$$

According to (19) and (42), for any $t \geq T_{7}$, we get that

$$
x_{1}(t) \geq \frac{\alpha_{1}^{L}\left(1-e^{-\gamma_{1}^{L} \tau_{1}}\right) m_{2}^{(2)}}{\gamma_{1}^{M}}:=m_{1}^{(2)} .
$$

For any $t \geq T_{7}$, it follows from the fourth equation of model system (4) that

$$
\dot{y}_{2}(t) \geq \alpha_{2}^{L} e^{-\gamma_{2}^{M} \tau_{2}} y_{2}\left(t-\tau_{2}\right)-\beta_{2}^{M} y_{2}^{2}(t)-c_{2}^{M} M_{2}^{(2)} y_{2}(t) .
$$

Based on Lemma 3 and (45), there exists $T_{8}>T_{7}$ and, for any $t \geq T_{8}$ and sufficiently small $\epsilon>0$,

$$
y_{2}(t) \geq \frac{\alpha_{2}^{L} e^{-\gamma_{2}^{M} \tau_{2}}-c_{2}^{M} M_{2}^{(2)}}{\beta_{2}^{M}}-\epsilon:=m_{4}^{(2)}
$$

holds provided that

$$
\alpha_{2}^{L} e^{-\gamma_{2}^{M} \tau_{2}}>c_{2}^{M} M_{2}^{(2)}
$$

According to (23) and (46), it can be obtained that

$$
y_{1}(t) \geq \frac{\alpha_{2}^{L} m_{4}^{(2)}\left(1-e^{-\gamma_{2}^{L} \tau_{2}}\right)}{\gamma_{2}^{M}}:=m_{3}^{(2)} .
$$

By using simple computation, it is easy to show that six inequalities (27), (31), (35), (39), (43), and (47) hold if the following two inequalities $\alpha_{1}^{L} \beta_{2}^{L}>c_{1}^{M} \alpha_{2}^{M}$ and $\alpha_{2}^{L} \beta_{1}^{L}>c_{2}^{M} \alpha_{1}^{M}$ hold.

Furthermore, eight sequences will be obtained by repeating the discussion in this manner, which are given as follows:

$$
\begin{aligned}
& M_{1}^{(n+1)}=\frac{\alpha_{1}^{M} M_{2}^{(n+1)}\left(1-e^{-\gamma_{1}^{M} \tau_{1}}\right)}{\gamma_{1}^{L}}, \\
& M_{2}^{(n+1)}=\frac{\alpha_{1}^{M} e^{-\gamma_{1}^{L} \tau_{1}}-c_{1}^{L} m_{4}^{(n)}}{\beta_{1}^{L}+\rho^{L}\left(m_{4}^{(n)}\right)^{2}}+\epsilon, \\
& M_{3}^{(n+1)}=\frac{\alpha_{2}^{M} M_{4}^{(n+1)}\left(1-e^{-\gamma_{2}^{M} \tau_{2}}\right)}{\gamma_{2}^{L}}, \\
& M_{4}^{(n+1)}=\frac{\alpha_{2}^{M} e^{-\gamma_{2}^{L} \tau_{2}}-c_{2}^{L} m_{2}^{(n)}+\epsilon,}{\beta_{2}^{L}} \\
& m_{1}^{(n+1)}=\frac{\alpha_{1}^{L}\left(1-e^{-\gamma_{1}^{L} \tau_{1}}\right) m_{2}^{(n+1)}}{\gamma_{1}^{M}},
\end{aligned}
$$

$$
\begin{gathered}
m_{2}^{(n+1)}=\frac{\alpha_{1}^{L} e^{-\gamma_{1}^{M} \tau_{1}}-c_{1}^{M} M_{4}^{(n+1)}}{\beta_{1}^{M}+\rho^{M}\left(M_{4}^{(n+1)}\right)^{2}}-\epsilon, \\
m_{3}^{(n+1)}=\frac{\alpha_{2}^{L} m_{4}^{(n+1)}\left(1-e^{-\gamma_{2}^{L} \tau_{2}}\right)}{\gamma_{2}^{M}}, \\
m_{4}^{(n+1)}=\frac{\alpha_{2}^{L} e^{-\gamma_{2}^{M} \tau_{2}}-c_{2}^{M} M_{2}^{(n+1)}}{\beta_{2}^{M}}-\epsilon .
\end{gathered}
$$

It is easy to show that $M_{i}^{(n)}>0$ and the sequences $\left\{M_{i}^{(n)}\right\}$ $(i=1,2,3,4)$ are decreasing as $n$ increases, which implies that $\lim _{n \rightarrow \infty} M_{i}^{(n)}=M_{i}^{*}$ exists; furthermore, it is easy to show that $m_{i}^{(n)}<M_{i}^{(n)}$ and the sequences $\left\{m_{i}^{(n)}\right\}(i=1,2,3,4)$ are increasing as $n$ increases, which implies that $\lim _{n \rightarrow \infty} m_{i}^{(n)}=$ $m_{i}^{*}$ exists. Consequently, it follows from (49) that

$$
\begin{array}{ll}
M_{1}^{*}=\frac{\alpha_{1}^{M} M_{2}^{*}\left(1-e^{-\gamma_{1}^{M} \tau_{1}}\right)}{\gamma_{1}^{L}}, & M_{2}^{*}=\frac{\alpha_{1}^{M} e^{-\gamma_{1}^{L} \tau_{1}}-c_{1}^{L} m_{4}^{*}}{\beta_{1}^{L}+\rho^{L} m_{4}^{* 2}}, \\
M_{3}^{*}=\frac{\alpha_{2}^{M} M_{4}^{*}\left(1-e^{-\gamma_{2}^{M} \tau_{2}}\right)}{\gamma_{2}^{L}}, & M_{4}^{*}=\frac{\alpha_{2}^{M} e^{-\gamma_{2}^{L} \tau_{2}}-c_{2}^{L} m_{2}^{*}}{\beta_{2}^{L}}, \\
m_{1}^{*}=\frac{\alpha_{1}^{L}\left(1-e^{-\gamma_{1}^{L} \tau_{1}}\right) m_{2}^{*}}{\gamma_{1}^{M}}, & m_{2}^{*}=\frac{\alpha_{1}^{L} e^{-\gamma_{1}^{M} \tau_{1}}-c_{1}^{M} M_{4}^{*}}{\beta_{1}^{M}+\rho^{M} M_{4}^{* 2}}, \\
m_{3}^{*}=\frac{\alpha_{2}^{L} m_{4}^{*}\left(1-e^{-\gamma_{2}^{L} \tau_{2}}\right)}{\gamma_{2}^{M}}, & m_{4}^{*}=\frac{\alpha_{2}^{L} e^{-\gamma_{2}^{M} \tau_{2}}-c_{2}^{M} M_{2}^{*}}{\beta_{2}^{M}} .
\end{array}
$$

Based on Definition 5 and (50), it can be concluded that model system (4) is persistent if $\alpha_{1}^{L} \beta_{2}^{L}>c_{1}^{M} \alpha_{2}^{M}$ and $\alpha_{2}^{L} \beta_{1}^{L}>$ $c_{2}^{M} \alpha_{1}^{M}$ hold.

\subsection{Existence of Positive Periodic Solutions}

Definition 8 (see [31]). Let $L:$ Dom $L \subset X \rightarrow Y$ be a linear mapping and let $N: X \rightarrow Y$ be a continuous mapping, where $X$ and $Y$ are real Banach spaces. If $\operatorname{dimKer} L=$ codim $\operatorname{Im} L<+\infty$ and $\operatorname{Im} L$ is closed in $Y$, then $L$ is called a Fredholm mapping of index zero.

If $L$ is Fredholm mapping of index zero and there exist continuous projectors $P: X \rightarrow X$ and $Q: Y \rightarrow Y$ such that

$$
\operatorname{Im} P=\operatorname{Ker} L, \quad \operatorname{Im} L=\operatorname{Ker} Q=\operatorname{Im}(I-Q),
$$

then restriction $L_{p}$ of $L$ to $\operatorname{Dom} L \cap \operatorname{Ker} P:(I-P) X \rightarrow \operatorname{Im} L$ is invertible.

Definition 9 (see [31]). Denote the inverse of $L_{p}$ by $K_{p}$. Supposing that $\Omega$ is an open bounded subset of $X$, if $Q N(\bar{\Omega})$ is bounded and $K_{p}(I-Q) N: \Omega \rightarrow X$ is compact, then the mapping $N$ is called $L$-compact on $\bar{\Omega}$. Since $\operatorname{Im} Q$ is 
isomorphic to $\operatorname{Ker} L$, there exists an isomorphism $J: \operatorname{Im} Q \rightarrow$ Ker $L$.

Lemma 10 (see [31]). Let $\Omega \subset X$ be an open bounded set, let $L$ be a Fredholm mapping of index zero, and let $N$ be L-compact on $\bar{\Omega}$. If the following three conditions hold:

(i) $L x \neq \lambda N x$ for any $\lambda \in(0,1)$ and $x \in \partial \Omega \cap \operatorname{Dom} L$,

(ii) $Q N x \neq 0$ for any $x \in \partial \Omega \cap \operatorname{Ker} L$,

(iii) $\operatorname{deg}\{J Q N, \Omega \cap \operatorname{Ker} L, 0\} \neq 0$,

then $L x=N x$ has at least one solution in $\bar{\Omega} \cap \operatorname{Dom} L$.

Theorem 11. If $\alpha_{1}^{L} \beta_{2}^{L} e^{-\gamma_{2}^{M} \tau_{1}}>\alpha_{2}^{M} c_{1}^{M} e^{-\gamma_{2}^{L} \tau_{2}}, \alpha_{2}^{L} \beta_{1}^{L} e^{-\gamma_{2}^{M} \tau_{2}}>$ $\alpha_{1}^{M} c_{2}^{M} e^{-\gamma_{1}^{L} \tau_{2}}$, then model system (4) with initial conditions (5) and (6) has at least one positive w-periodic solution.

Proof. Consider the subsystem of model system (4):

$$
\begin{aligned}
\dot{x}_{2}(t)= & \alpha_{1}\left(t-\tau_{1}\right) e^{-\int_{t-\tau_{1}}^{t} \gamma_{1}(s) \mathrm{d} s} x_{2}\left(t-\tau_{1}\right)-\beta_{1}(t) x_{2}^{2}(t) \\
& -c_{1}(t) x_{2}(t) y_{2}(t)-\rho(t) x_{2}^{2}(t) y_{2}^{2}(t) \\
\dot{y}_{2}(t)= & \alpha_{2}\left(t-\tau_{2}\right) e^{-\int_{t-\tau_{2}}^{t} \gamma_{2}(s) \mathrm{d} s} y_{2}\left(t-\tau_{2}\right) \\
& -\beta_{2}(t) y_{2}^{2}(t)-c_{2}(t) x_{2}(t) y_{2}(t) .
\end{aligned}
$$

Let $u_{1}(t)=\ln \left[x_{2}(t)\right], u_{2}(t)=\ln \left[y_{2}(t)\right]$. that

By substituting $u_{1}(t)$ and $u_{2}(t)$ into (52), it can be obtained

$$
\begin{aligned}
\dot{u}_{1}(t)= & \alpha_{1}\left(t-\tau_{1}\right) e^{-\int_{t-\tau_{1}}^{t} \gamma_{1}(s) \mathrm{d} s} e^{u_{1}\left(t-\tau_{1}\right)-u_{1}(t)} \\
& -\beta_{1}(t) e^{u_{1}(t)}-c_{1}(t) e^{u_{2}(t)}-\rho(t) e^{u_{1}(t)+2 u_{2}(t)} \\
\dot{u}_{2}(t)= & \alpha_{2}\left(t-\tau_{2}\right) e^{-\int_{t-\tau_{2}}^{t} \gamma_{2}(s) \mathrm{d} s} e^{u_{2}\left(t-\tau_{2}\right)-u_{2}(t)} \\
& -\beta_{2}(t) e^{u_{2}(t)}-c_{2}(t) e^{u_{1}(t)}
\end{aligned}
$$

It should be noted that if model system (53) has one $\omega$-periodic solution $\left(u_{1}^{*}(t), u_{2}^{*}(t)\right)^{T}$, then $\left(x_{2}^{*}(t), y_{2}^{*}(t)\right)^{T}=$ $\left(e^{u_{1}^{*}(t)}, e^{u_{2}^{*}(t)}\right)^{T}$ is a positive $\omega$-periodic solution of model system (52).

In order to utilize Lemma 10 in a straightforward manner, we define

$$
\begin{aligned}
& X=Y=\left\{\left(u_{1}(t), u_{2}(t)\right)^{T}\right. \\
& \left.\quad \in C\left(\mathbb{R}, \mathbb{R}^{2}\right): u_{i}(t+\omega)=u_{i}(t), i=1,2\right\}, \\
& \left\|\left(u_{1}(t), u_{2}(t)\right)^{T}\right\|=\max _{t \in[0, \omega]}\left|u_{1}(t)\right|+\max _{t \in[0, \omega]}\left|u_{2}(t)\right|,
\end{aligned}
$$

where $|\cdot|$ denotes the Euclidean norm; it is easy to show that both $X$ and $Y$ are Banach spaces with the norm $\|\cdot\|$; then define

$$
\begin{gathered}
\operatorname{Dom} L \cap X \rightarrow X, \\
L\left(u_{1}(t), u_{2}(t)\right)^{T}=\left(\frac{\mathrm{d} u_{1}(t)}{\mathrm{d} t}, \frac{\mathrm{d} u_{2}(t)}{\mathrm{d} t}\right)^{T},
\end{gathered}
$$

where $\operatorname{Dom} L=\left\{\left(u_{1}(t), u_{2}(t)\right)^{T} \in C\left(\mathbb{R}, \mathbb{R}^{2}\right)\right\}, N\left[\begin{array}{l}u_{1} \\ u_{2}\end{array}\right]=$ $\left[\begin{array}{l}f_{1}(t) \\ f_{2}(t)\end{array}\right]$, and

$$
\begin{aligned}
f_{1}(t)= & \alpha_{1}\left(t-\tau_{1}\right) e^{-\int_{t-\tau_{1}}^{t} \gamma_{1}(s) \mathrm{d} s} e^{u_{1}\left(t-\tau_{1}\right)-u_{1}(t)} \\
& -\beta_{1}(t) e^{u_{1}(t)}-c_{1}(t) e^{u_{2}(t)}-\rho(t) e^{u_{1}(t)+2 u_{2}(t)}, \\
f_{2}(t)= & \alpha_{2}\left(t-\tau_{2}\right) e^{-\int_{t-\tau_{2}}^{t} \gamma_{2}(s) \mathrm{d} s} e^{u_{2}\left(t-\tau_{2}\right)-u_{2}(t)} \\
& -\beta_{2}(t) e^{u_{2}(t)}-c_{2}(t) e^{u_{1}(t)} .
\end{aligned}
$$

Furthermore, we define

$$
P\left[\begin{array}{l}
u_{1} \\
u_{2}
\end{array}\right]=Q\left[\begin{array}{l}
u_{1} \\
u_{2}
\end{array}\right]=\left[\begin{array}{l}
\frac{1}{\omega} \int_{0}^{\omega} u_{1}(t) \mathrm{d} t \\
\frac{1}{\omega} \int_{0}^{\omega} u_{2}(t) \mathrm{d} t
\end{array}\right], \quad\left[\begin{array}{l}
u_{1} \\
u_{2}
\end{array}\right] \in X=Y .
$$

According to the above definitions, it is not difficult to verify that $\operatorname{Ker} L=\left\{x \mid x \in X, x=h, h \in \mathbb{R}^{2}\right\}$, $\operatorname{Im} L=\left\{y \in Y \mid \int_{0}^{\omega} y(t) \mathrm{d} t=0\right\}$ are closed in $Y, \operatorname{dim} \operatorname{Ker} L=$ codim Im $L=2$, and both $P$ and $Q$ are continuous projectors such that $\operatorname{Im} P=\operatorname{Ker} L$ and $\operatorname{Ker} Q=\operatorname{Im} L=\operatorname{Im}(I-Q)$.

Based on the above analysis, it can be obtained that $L$ is a Fredholm mapping of index zero.

Furthermore, the inverse $K_{p}: \operatorname{Im} L \rightarrow \operatorname{Dom} L \cap \operatorname{Ker} P$ of $L_{p}$ exists and takes the following form:

$$
K_{p}(y)=\int_{0}^{t} y(s) \mathrm{d} s-\frac{1}{\omega} \int_{0}^{\omega} \int_{0}^{t} y(s) \mathrm{d} s \mathrm{~d} t .
$$

Hence, $Q N: X \rightarrow Y$ and $K_{p}(I-Q) N: X \rightarrow X$ can be defined as follows, respectively,

$$
\begin{aligned}
Q N x= & {\left[\begin{array}{c}
\frac{1}{\omega} \int_{0}^{\omega} f_{1}(t) \mathrm{d} t \\
\frac{1}{\omega} \int_{0}^{\omega} f_{2}(t) \mathrm{d} t
\end{array}\right], } \\
K_{p}(I-Q) N x= & \int_{0}^{t} N x(s) \mathrm{d} s-\frac{1}{\omega} \int_{0}^{\omega} \int_{0}^{t} N x(s) \mathrm{d} s \mathrm{~d} t \\
& -\left(\frac{t}{\omega}-\frac{1}{2}\right) \int_{0}^{\omega} N x(s) \mathrm{d} s .
\end{aligned}
$$

It is easy to show that $Q N$ and $K_{p}(I-Q) N$ are continuous. In order to facilitate the proof based on Lemma 10, we also need to find an appropriate open and bounded subset $\Omega$, which can be found by the following two steps. 
Step 1. According to the operator equation $L x=\lambda N x$ for $\lambda \epsilon$ $(0,1)$, the upper and lower bound of $u_{1}(t)$ and $u_{2}(t)$ will be estimated as follows:

$$
\begin{aligned}
& \frac{\mathrm{d} u_{1}(t)}{\mathrm{d} t}=\lambda f_{1}(t), \\
& \frac{\mathrm{d} u_{2}(t)}{\mathrm{d} t}=\lambda f_{2}(t),
\end{aligned}
$$

where $f_{1}(t)$ and $f_{2}(t)$ have been defined in (56).

Suppose that $\left(u_{1}(t), u_{2}(t)\right)^{T} \in X$ is a solution of model system (60) for some $\lambda \in(0,1)$. By integrating (60) over the interval $[0, \omega]$, it can be obtained that

$$
\begin{aligned}
& \int_{0}^{\omega} \alpha_{1}\left(t-\tau_{1}\right) e^{-\int_{t-\tau_{1}}^{t} \gamma_{1}(s) \mathrm{d} s} e^{u_{1}\left(t-\tau_{1}\right)-u_{1}(t)} \mathrm{d} t \\
& \quad=\int_{0}^{\omega} \beta_{1}(t) e^{u_{1}(t)}+c_{1}(t) e^{u_{2}(t)}+\rho(t) e^{u_{1}(t)+2 u_{2}(t)} \mathrm{d} t \\
& \int_{0}^{\omega} \alpha_{2}\left(t-\tau_{2}\right) e^{-\int_{t-\tau_{2}}^{t} \gamma_{2}(s) \mathrm{d} s} e^{u_{2}\left(t-\tau_{2}\right)-u_{2}(t)} \mathrm{d} t \\
& \quad=\int_{0}^{\omega} \beta_{2}(t) e^{u_{2}(t)}+c_{2}(t) e^{u_{1}(t)} \mathrm{d} t
\end{aligned}
$$

Based on definition $\left(u_{1}(t), u_{2}(t)\right)^{T} \in X$, there exist $\xi_{i}, \eta_{i} \in$ $[0, \omega]$ such that

$$
u_{i}\left(\xi_{i}\right)=\min _{t \in[0, \omega]} u_{i}(t), \quad u_{i}\left(\eta_{i}\right)=\max _{t \in[0, \omega]} u_{i}(t), \quad i=1,2 .
$$

Multiplying the first equation of (60) by $e^{u_{1}(t)}$ and integrating it over $[0, \omega]$ give that

$$
\begin{gathered}
\int_{0}^{\omega} \alpha_{1}\left(t-\tau_{1}\right) e^{-\int_{t-\tau_{1}}^{t} \gamma_{1}(s) \mathrm{d} s} e^{u_{1}\left(t-\tau_{1}\right)} \mathrm{d} t \\
=\int_{0}^{\omega} \beta_{1}(t) e^{2 u_{1}(t)}+c_{1}(t) e^{u_{1}(t)+u_{2}(t)} \\
+\rho(t) e^{2\left(u_{1}(t)+u_{2}(t)\right)} \mathrm{d} t .
\end{gathered}
$$

It follows from (64) that

$$
\begin{aligned}
& \beta_{1}^{L} \int_{0}^{\omega} e^{2 u_{1}(t)} \mathrm{d} t+c_{1}^{L} \int_{0}^{\omega} e^{u_{1}(t)+u_{2}(t)} \mathrm{d} t \\
& \quad+\rho^{L} \int_{0}^{\omega} e^{2\left(u_{1}(t)+u_{2}(t)\right)} \mathrm{d} t \leq \alpha_{1}^{M} e^{-\gamma_{1}^{L} \tau_{1}} \int_{0}^{\omega} e^{u_{1}(t)} \mathrm{d} t .
\end{aligned}
$$

On the other hand, by using the inequality,

$$
\left(\int_{0}^{\omega} e^{u_{1}(t)} \mathrm{d} t\right)^{2} \leq \omega \int_{0}^{\omega} e^{2 u_{1}(t)} \mathrm{d} t .
$$

Based on (65) and (66), it can be obtained that

$$
\begin{aligned}
\beta_{1}^{L}\left(\int_{0}^{\omega} e^{u_{1}(t)} \mathrm{d} t\right)^{2} & \leq \omega \beta_{1}^{L} \int_{0}^{\omega} e^{2 u_{1}(t)} \mathrm{d} t \\
& \leq \omega \alpha_{1}^{M} e^{-\gamma_{1}^{L} \tau_{1}} \int_{0}^{\omega} e^{u_{1}(t)} \mathrm{d} t
\end{aligned}
$$

which derives that

$$
\beta_{1}^{L} \int_{0}^{\omega} e^{u_{1}(t)} \mathrm{d} t \leq \omega \alpha_{1}^{M} e^{-\gamma_{1}^{L} \tau_{1}}, \quad u_{1}\left(\xi_{1}\right) \leq \ln \frac{\alpha_{1}^{M} e^{-\gamma_{1}^{L} \tau_{1}}}{\beta_{1}^{L}} .
$$

It follows from (60) and (68) that

$$
\int_{0}^{\omega}\left|u_{1}^{\prime}(t)\right| \mathrm{d} t<2 \int_{0}^{\omega} \beta_{1}(t) e^{u_{1}(t)} \leq \frac{2 \omega \alpha_{1}^{M} \beta_{1}^{M} e^{-\gamma_{1}^{L} \tau_{1}}}{\beta_{1}^{L}} .
$$

According to (68) and (69), it can be obtained that

$$
\begin{aligned}
u_{1}(t) & \leq u_{1}\left(\xi_{1}\right)+\int_{0}^{\omega}\left|u_{1}^{\prime}(t)\right| \mathrm{d} t \\
& \leq \ln \frac{\alpha_{1}^{M} e^{-\gamma_{1}^{L} \tau_{1}}}{\beta_{1}^{L}}+\frac{2 \omega \alpha_{1}^{M} \beta_{1}^{M} e^{-\gamma_{1}^{L} \tau_{1}}}{\beta_{1}^{L}} .
\end{aligned}
$$

Multiplying the second equation of (60) by $e^{u_{2}(t)}$ and integrating it over $[0, \omega]$ give that

$$
\begin{aligned}
\int_{0}^{\omega} \alpha_{2}\left(t-\tau_{2}\right) e^{-\int_{t-\tau_{2}}^{t} \gamma_{2}(s) \mathrm{d} s} e^{u_{2}\left(t-\tau_{2}\right)} \mathrm{d} t \\
\quad=\int_{0}^{\omega} \beta_{2}(t) e^{2 u_{2}(t)}+c_{2}(t) e^{u_{1}(t)+u_{2}(t)} \mathrm{d} t .
\end{aligned}
$$

It follows from (71) that

$$
\begin{gathered}
\beta_{2}^{L} \int_{0}^{\omega} e^{2 u_{2}(t)} \mathrm{d} t+c_{2}^{L} \int_{0}^{\omega} e^{u_{1}(t)+u_{2}(t)} \mathrm{d} t \\
\leq \alpha_{2}^{M} e^{-\gamma_{2}^{L} \tau_{2}} \int_{0}^{\omega} e^{u_{2}(t)} \mathrm{d} t .
\end{gathered}
$$

Based on (66) and (72), it can be obtained that

$$
\beta_{2}^{L}\left(\int_{0}^{\omega} e^{u_{1}(t)} \mathrm{d} t\right)^{2} \leq \omega \alpha_{2}^{M} e^{-\gamma_{2}^{L} \tau_{2}} \int_{0}^{\omega} e^{u_{2}(t)} \mathrm{d} t
$$

which derives that

$\int_{0}^{\omega} e^{u_{2}(t)} \mathrm{d} t \leq \frac{\omega \alpha_{2}^{M} e^{-\gamma_{2}^{L} \tau_{2}}}{\beta_{2}^{L}}, \quad u_{2}\left(\xi_{2}\right) \leq \ln \frac{\alpha_{2}^{M} e^{-\gamma_{2}^{L} \tau_{2}}}{\beta_{2}^{L}}$.

It follows from (60) and (74) that

$\int_{0}^{\omega}\left|u_{2}^{\prime}(t)\right| \mathrm{d} t<2 \int_{0}^{\omega} \beta_{2}(t) e^{u_{2}(t)} \mathrm{d} t \leq \frac{2 \omega \alpha_{2}^{M} \beta_{2}^{M} e^{-\gamma_{2}^{L} \tau_{2}}}{\beta_{2}^{L}}$.

According to (74) and (75), it can be obtained that

$$
\begin{aligned}
u_{2}(t) & \leq u_{2}\left(\xi_{2}\right)+\int_{0}^{\omega}\left|u_{2}^{\prime}(t)\right| \mathrm{d} t \\
& \leq \ln \frac{\alpha_{2}^{M} e^{-\gamma_{2}^{L} \tau_{2}}}{\beta_{2}^{L}}+\frac{2 \omega \alpha_{2}^{M} \beta_{2}^{M} e^{-\gamma_{2}^{L} \tau_{2}}}{\beta_{2}^{L}} .
\end{aligned}
$$


It should be noted that

$$
\begin{aligned}
& \int_{0}^{\omega} \alpha_{1}(t) e^{-\int_{t}^{t+\tau_{1}} \gamma_{1}(s) \mathrm{d} s} e^{u_{1}(t)} \mathrm{d} t \\
& \quad=\int_{0}^{\omega} \alpha_{1}\left(t-\tau_{1}\right) e^{-\int_{t-\tau_{1}}^{t} \gamma_{1}(s) \mathrm{d} s} e^{u_{1}\left(t-\tau_{1}\right)} \mathrm{d} t .
\end{aligned}
$$

Based on (64), it can be obtained that

$$
\begin{gathered}
\left(\alpha_{1}^{L} e^{-\gamma_{2}^{M} \tau_{1}}-\frac{c_{1}^{M} \alpha_{2}^{M} e^{-\gamma_{2}^{L} \tau_{2}}}{\beta_{2}^{L}}\right) \int_{0}^{\omega} e^{u_{1}(t)} \mathrm{d} t \\
\leq\left(\beta_{1}^{M}+\frac{2 \rho^{M} \alpha_{2}^{M} e^{-\gamma_{2}^{L} \tau_{2}}}{\beta_{2}^{L}}\right) \int_{0}^{\omega} e^{2 u_{1}(t)} \mathrm{d} t,
\end{gathered}
$$

which derives that

$$
\begin{gathered}
\int_{0}^{\omega} e^{u_{1}(t)} \mathrm{d} t \geq \frac{\omega\left(\alpha_{1}^{L} \beta_{2}^{L} e^{-\gamma_{2}^{M} \tau_{1}}-\alpha_{2}^{M} c_{1}^{M} e^{-\gamma_{2}^{L} \tau_{2}}\right)}{\beta_{1}^{M} \beta_{2}^{L}+2 \alpha_{2}^{M} \rho^{M} e^{-\gamma_{2}^{L} \tau_{2}}}, \\
u_{1}\left(\eta_{1}\right) \geq \ln \frac{\alpha_{1}^{L} \beta_{2}^{L} e^{-\gamma_{2}^{M} \tau_{1}}-\alpha_{2}^{M} c_{1}^{M} e^{-\gamma_{2}^{L} \tau_{2}}}{\beta_{1}^{M} \beta_{2}^{L}+2 \alpha_{2}^{M} \rho^{M} e^{-\gamma_{2}^{L} \tau_{2}}}
\end{gathered}
$$

hold provided that $\alpha_{1}^{L} \beta_{2}^{L} e^{-\gamma_{2}^{M} \tau_{1}}>\alpha_{2}^{M} c_{1}^{M} e^{-\gamma_{2}^{L} \tau_{2}}$.

According to (69) and (79), it can be obtained that

$$
\begin{aligned}
u_{1}(t) & \geq u_{1}\left(\eta_{1}\right)-\int_{0}^{\omega}\left|u_{1}^{\prime}(t)\right| \mathrm{d} t \\
& \geq \ln \frac{\alpha_{1}^{L} \beta_{2}^{L} e^{-\gamma_{2}^{M} \tau_{1}}-\alpha_{2}^{M} c_{1}^{M} e^{-\gamma_{2}^{L} \tau_{2}}}{\beta_{1}^{M} \beta_{2}^{L}+2 \alpha_{2}^{M} \rho^{M} e^{-\gamma_{2}^{L} \tau_{2}}}-\frac{2 \omega \alpha_{1}^{M} \beta_{1}^{M} e^{-\gamma_{2}^{L} \tau_{2}}}{\beta_{1}^{L}} .
\end{aligned}
$$
then

By virtue of (70) and (80), if $\alpha_{1}^{L} \beta_{2}^{L} e^{-\gamma_{2}^{M} \tau_{1}}>\alpha_{2}^{M} c_{1}^{M} e^{-\gamma_{2}^{L} \tau_{2}}$, $\max _{t \in[0, \omega]}\left|u_{1}(t)\right|$

$$
<\max \left\{\begin{array}{c}
\left|\ln \frac{\alpha_{1}^{M} e^{-\gamma_{1}^{L} \tau_{1}}}{\beta_{1}^{L}}\right|+\frac{2 \omega \alpha_{1}^{M} \beta_{1}^{M} e^{-\gamma_{1}^{L} \tau_{1}}}{\beta_{1}^{L}}, \\
\left|\ln \frac{\alpha_{1}^{L} \beta_{2}^{L} e^{-\gamma_{2}^{M} \tau_{1}}-\alpha_{2}^{M} c_{1}^{M} e^{-\gamma_{2}^{L} \tau_{2}}}{\beta_{1}^{M} \beta_{2}^{L}+2 \alpha_{2}^{M} \rho^{M} e^{-\gamma_{2}^{L} \tau_{2}}}\right|+\frac{2 \omega \alpha_{1}^{M} \beta_{1}^{M} e^{-\gamma_{1}^{L} \tau_{1}}}{\beta_{1}^{L}}
\end{array}\right\}
$$

$:=H_{1}$.

Similarly, it is easy to show that

$$
\begin{aligned}
& \int_{0}^{\omega} \alpha_{2}(t) e^{-\int_{t}^{t+\tau_{2}} \gamma_{2}(s) \mathrm{d} s} e^{u_{2}(t)} \mathrm{d} t \\
& \quad=\int_{0}^{\omega} \alpha_{2}\left(t-\tau_{2}\right) e^{-\int_{t-\tau_{2}}^{t} \gamma_{2}(s) \mathrm{d} s} e^{u_{2}\left(t-\tau_{2}\right)} \mathrm{d} t,
\end{aligned}
$$

which derives that

$$
u_{2}\left(\eta_{2}\right) \geq \ln \frac{\alpha_{2}^{L} \beta_{1}^{L} e^{-\gamma_{2}^{M} \tau_{2}}-\alpha_{1}^{M} c_{2}^{M} e^{-\gamma_{1}^{L} \tau_{1}}}{\beta_{1}^{L} \beta_{2}^{M}}
$$

holds provided that $\alpha_{2}^{L} \beta_{1}^{L} e^{-\gamma_{2}^{M} \tau_{2}}>\alpha_{1}^{M} c_{2}^{M} e^{-\gamma_{1}^{L} \tau_{1}}$.
According to (75) and (83), it is derived that

$$
\begin{aligned}
u_{2}(t) & \geq u_{2}\left(\eta_{2}\right)-\int_{0}^{\omega}\left|u_{2}^{\prime}(t)\right| \mathrm{d} t \\
& \geq \ln \frac{\alpha_{2}^{L} \beta_{1}^{L} e^{-\gamma_{2}^{M} \tau_{2}}-\alpha_{1}^{M} c_{2}^{M} e^{-\gamma_{1}^{L} \tau_{1}}}{\beta_{1}^{L} \beta_{2}^{M}}-\frac{2 \omega \alpha_{2}^{M} \beta_{2}^{M} e^{-\gamma_{2}^{L} \tau_{2}}}{\beta_{2}^{L}} .
\end{aligned}
$$

By virtue of (76) and (84), if $\alpha_{2}^{L} \beta_{1}^{L} e^{-\gamma_{2}^{M} \tau_{2}}>\alpha_{1}^{M} c_{2}^{M} e^{-\gamma_{1}^{L} \tau_{2}}$, then

$$
\max _{t \in[0, \omega]}\left|u_{2}(t)\right|
$$$$
<\max \left\{\begin{array}{c}
\left|\ln \frac{\alpha_{2}^{M} e^{-\gamma_{2}^{L} \tau_{2}}}{\beta_{2}^{L}}\right|+\frac{2 \omega \alpha_{2}^{M} \beta_{2}^{M} e^{-\gamma_{2}^{L} \tau_{2}}}{\beta_{2}^{L}}, \\
\left|\ln \frac{\alpha_{2}^{L} \beta_{1}^{L} e^{-\gamma_{2}^{M} \tau_{2}}-\alpha_{1}^{M} c_{2}^{M} e^{-\gamma_{1}^{L} \tau_{1}}}{\beta_{1}^{L} \beta_{2}^{M}}\right|+\frac{2 \omega \alpha_{2}^{M} \beta_{2}^{M} e^{-\gamma_{2}^{L} \tau_{2}}}{\beta_{2}^{L}}
\end{array}\right\}
$$

$:=H_{2}$.

It is obvious that $H_{1}$ and $H_{2}$ in (81) and (85) are independent of $\lambda$.

Step 2. In order to construct an appropriate open and bounded subset $\Omega$, denote $H=H_{1}+H_{2}+H_{0}$, where $H_{0}$ is sufficiently large such that the unique solution $\left(u^{*}, v^{*}\right)^{T}$ of the following algebraic equations:

$$
\frac{1}{\omega} \int_{0}^{\omega} f_{1}(t) \mathrm{d} t=0, \quad \frac{1}{\omega} \int_{0}^{\omega} f_{2}(t) \mathrm{d} t=0,
$$

satisfies $\left\|\left(u^{*}, v^{*}\right)^{T}\right\|=\left|u^{*}\right|+\left|v^{*}\right|<H$.

Select $\Omega=\left\{\left(u_{1}(t), u_{2}(t)\right)^{T} \in X:\left\|\left(u_{1}, u_{2}\right)^{T}\right\|<H\right\}$, which implies that condition (i) of Lemma 10 holds.

When $\left(u_{1}(t), u_{2}(t)\right)^{T} \in \partial \Omega \cap \operatorname{Ker} L=\partial \Omega \cap \mathbb{R}^{2},\left(u_{1}, u_{2}\right)^{T}$ is a constant vector in $\mathbb{R}^{2}$ with $\left|u_{1}\right|+\left|u_{2}\right|=H$. Consequently, it can be concluded that

$$
Q N\left[\begin{array}{l}
u_{1} \\
u_{2}
\end{array}\right]=\left[\begin{array}{l}
\frac{1}{\omega} \int_{0}^{\omega} f_{1}(t) \mathrm{d} t \\
\frac{1}{\omega} \int_{0}^{\omega} f_{2}(t) \mathrm{d} t
\end{array}\right] \neq\left[\begin{array}{l}
0 \\
0
\end{array}\right],
$$

which implies that condition (ii) of Lemma 10 is satisfied.

Take $J=I: \operatorname{Im} Q \rightarrow \operatorname{Ker} L,\left(u_{1}, u_{2}\right)^{T} \rightarrow\left(u_{1}, u_{2}\right)^{T}$. It follows from straightforward computation that

$$
\operatorname{deg}\left(J Q N\left(u_{1}, u_{2}\right)^{T}, \Omega \cap \operatorname{Ker} L,(0,0)^{T}\right)=1,
$$

where $\left(u_{1}^{*}, u_{2}^{*}\right)$ is the unique solution of (86). Hence, the condition (iii) of Lemma 10 holds.

Furthermore, it is easy to see that the set $\left\{K_{p}(I-Q) N x \mid\right.$ $x \in \bar{\Omega}\}$ is equicontinuous and uniformly bounded. By using the Arzela-Ascoli theorem [31], it can be shown that $K_{p}(I-$ Q) $N: \bar{\Omega} \rightarrow X$ is compact and $N$ is $L$-compact. 
Consequently, all conditions (i)-(iii) of Lemma 10 hold for $\Omega$. It follows from Lemma 10 that model system (53) has at least one $\omega$-periodic solution $\left(u_{1}^{*}(t), u_{2}^{*}(t)\right)^{T}$, and model system (52) has at least one $\omega$-periodic solution $\left(x_{2}^{*}(t), y_{2}^{*}(t)\right)^{T}=$ $\left(e^{u_{1}^{*}(t)}, e^{u_{2}^{*}(t)}\right)^{T}$.

Let $\left(x_{2}^{*}(t), y_{2}^{*}(t)\right)^{T}$ be a positive $\omega$-periodic solution of model system (52); it follows from (19) and (23) that

$$
\begin{aligned}
& x_{1}^{*}(t)=e^{-\int_{0}^{t} \gamma_{1}(s) \mathrm{d} s} \int_{t-\tau_{1}}^{t} \alpha_{1}(s) e^{\int_{0}^{s} \gamma_{1}(m) \mathrm{d} m} x_{2}^{*}(s) \mathrm{d} s, \\
& y_{1}^{*}(t)=e^{-\int_{0}^{t} \gamma_{2}(s) \mathrm{d} s} \int_{t-\tau_{2}}^{t} \alpha_{2}(s) e^{\int_{0}^{s} \gamma_{2}(m) \mathrm{d} m} y_{2}^{*}(s) \mathrm{d} s
\end{aligned}
$$

are $\omega$-periodic continuous function.

Based on the above analysis, if the following two inequalities hold:

$$
\alpha_{1}^{L} \beta_{2}^{L} e^{-\gamma_{2}^{M} \tau_{1}}>\alpha_{2}^{M} c_{1}^{M} e^{-\gamma_{2}^{L} \tau_{2}}, \quad \alpha_{2}^{L} \beta_{1}^{L} e^{-\gamma_{2}^{M} \tau_{2}}>\alpha_{1}^{M} c_{2}^{M} e^{-\gamma_{1}^{L} \tau_{2}},
$$

then model system (4) with initial conditions (5) and (6) has at least one positive $\omega$-periodic solution $\left(x_{1}^{*}(t)\right.$, $\left.x_{2}^{*}(t), y_{1}^{*}(t), y_{2}^{*}(t)\right)^{T}$.

\subsection{Global Stability Analysis}

Theorem 12. If $\liminf _{t \rightarrow+\infty} G_{k}(t)>0, k=1,2$, then model system (4) with initial conditions (5) and (6) has a unique positive $\omega$-periodic globally stable solution $\left(x_{1}^{*}(t)\right.$, $\left.x_{2}^{*}(t), y_{1}^{*}(t), y_{2}^{*}(t)\right)^{T}$, where

$$
\begin{gathered}
G_{1}=-\alpha_{1}(t) e^{-\int_{t}^{t+\tau_{1}} \gamma_{1}(m) \mathrm{d} m}-2 c_{1}(t) M_{4}^{*} \\
+2 \beta_{1}(t) m_{2}^{*}-8 M_{2}^{*} M_{4}^{* 2} \rho(t), \\
G_{2}(t)=-q \alpha_{2}(t) e^{-\int_{t}^{t+\tau_{2}} \gamma_{2}(m) \mathrm{d} m}+2 q \beta_{2}(t) m_{4}^{*}+2 q c_{1}(t) m_{2}^{*},
\end{gathered}
$$

and $q$ is a positive constant and $m_{i}, M_{i}, i=1,2,3,4$, have been defined in (50).

Proof. Suppose that $\left(x_{1}^{*}(t), x_{2}^{*}(t), y_{1}^{*}(t), y_{2}^{*}(t)\right)^{T}$ is a positive $\omega$-periodic solution of model system (4) with initial conditions (5) and (6).

Construct a Lyapunov functional as follows:

$$
\begin{aligned}
V_{1}(t)=\left|x_{2}(t)-x_{2}^{*}(t)\right|+\int_{t-\tau_{1}}^{t} & \alpha_{1}(s) e^{-\int_{s}^{s+\tau_{1}} \gamma_{1}(s) \mathrm{d} m} \\
& \times\left|x_{2}(s)-x_{2}^{*}(s)\right| \mathrm{d} s .
\end{aligned}
$$

By calculating the upper right derivative of $V_{1}(t)$ along the positive $\omega$-periodic solution of model system (4), it can be obtained that

$$
\begin{aligned}
& D^{+} V_{1}(t)= \operatorname{sgn}\left[x_{2}(t)-x_{2}^{*}(t)\right] \\
& \times\left\{\alpha_{1}\left(t-\tau_{1}\right) e^{-\int_{t-\tau_{1}}^{t} \gamma_{1}(s) \mathrm{d} s} x_{2}\left(t-\tau_{1}\right)\right. \\
& \quad-\alpha_{1}\left(t-\tau_{1}\right) e^{-\int_{t-\tau_{1}}^{t} \gamma_{1}(s) \mathrm{d} s} x_{2}^{*}\left(t-\tau_{1}\right)
\end{aligned}
$$

$$
\begin{gathered}
-\beta_{1}(t) x_{2}^{2}(t)+\beta_{1}(t) x_{2}^{* 2}(t) \\
-c_{1}(t) x_{2}(t) y_{2}(t)+c_{1}(t) x_{2}^{*}(t) y_{2}^{*}(t) \\
\left.-\rho(t) x_{2}^{2}(t) y_{2}^{2}(t)+\rho(t) x_{2}^{* 2}(t) y_{2}^{* 2}(t)\right\} \\
+\alpha_{1}(t) e^{-\int_{t}^{t+\tau_{1}} \gamma_{1}(m) \mathrm{d} m}\left|x_{2}(t)-x_{2}^{*}(t)\right| \\
-\alpha_{1}\left(t-\tau_{1}\right) e^{-\int_{t-\tau_{1}}^{t} \gamma_{1}(m) \mathrm{d} m} \\
\times\left|x_{2}\left(t-\tau_{1}\right)-x_{2}^{*}\left(t-\tau_{1}\right)\right| \\
\leq-\beta_{1}(t)\left[x_{2}(t)+x_{2}^{*}(t)\right]\left|x_{2}(t)-x_{2}^{*}(t)\right| \\
+\alpha_{1}(t) e^{-\int_{t}^{t+\tau_{1}}} \gamma_{1}(m) \mathrm{d} m\left|x_{2}(t)-x_{2}^{*}(t)\right| \\
-c_{1}(t) \operatorname{sgn}\left[x_{2}(t)-x_{2}^{*}(t)\right] \\
\times\left[x_{2}(t) y_{2}(t)-x_{2}^{*}(t) y_{2}^{*}(t)\right] \\
-\rho(t) \operatorname{sgn}\left[x_{2}(t)-x_{2}^{*}(t)\right] \\
\times\left[x_{2}(t) y_{2}(t)+x_{2}^{*}(t) y_{2}^{*}(t)\right] \\
\times\left[x_{2}(t) y_{2}(t)-x_{2}^{*}(t) y_{2}^{*}(t)\right] \\
\leq-\left\{\beta_{1}(t)\left[\left(x_{2}(t)+x_{2}^{*}(t)\right)\right]\right. \\
+c_{1}(t)\left[y_{2}(t)-y_{2}^{*}(t)\right] \\
+\rho(t)\left(y_{2}(t)-y_{2}^{*}(t)\right) \\
\quad \times\left[\left(x_{2}(t)-x_{2}^{*}(t)\right) y_{2}(t)\right. \\
\left.\quad+\left(y_{2}(t)-y_{2}^{*}(t)\right) x_{2}^{*}(t)\right] \\
\left.-\alpha_{1}(t) e^{-\int_{t}^{t+\tau_{1}} \gamma_{1}(m) \mathrm{d} m}\right\}\left|x_{2}(t)-x_{2}^{*}(t)\right| \\
\\
+
\end{gathered}
$$

Similarly, construct another Lyapunov functional as follows:

$$
\begin{aligned}
V_{2}(t)= & \left|y_{2}(t)-y_{2}^{*}(t)\right| \\
& +\int_{t-\tau_{2}}^{t} \alpha_{2}(s) e^{-\int_{s}^{s+\tau_{2}} \gamma_{2}(s) \mathrm{d} m}\left|y_{2}(s)-y_{2}^{*}(s)\right| \mathrm{d} s .
\end{aligned}
$$

By calculating the upper right derivative of $V_{2}(t)$ along the positive $\omega$-periodic solution of model system (4), it can be obtained that

$$
\begin{aligned}
D^{+} V_{2}(t)=\operatorname{sgn}[ & \left.y_{2}(t)-y_{2}^{*}(t)\right] \\
\times\{ & \alpha_{2}\left(t-\tau_{2}\right) e^{-\int_{t-\tau_{2}}^{t} \gamma_{2}(s) \mathrm{d} s} \\
& \times\left[y_{2}\left(t-\tau_{2}\right)-y_{2}^{*}\left(t-\tau_{2}\right)\right] \\
& -\beta_{2}(t)\left[y_{2}(t)+y_{2}^{*}(t)\right]\left[y_{2}(t)-y_{2}^{*}(t)\right] \\
& \left.-c_{2}(t)\left[x_{2}(t) y_{2}(t)-x_{2}^{*}(t) y_{2}^{*}(t)\right]\right\}
\end{aligned}
$$




$$
\begin{aligned}
& +\alpha_{2}(t) e^{-\int_{t}^{t+\tau_{2}} \gamma_{2}(m) \mathrm{d} m}\left|y_{2}(t)-y_{2}^{*}(t)\right| \\
& -\alpha_{2}\left(t-\tau_{2}\right) e^{-\int_{t-\tau_{2}}^{t} \gamma_{2}(m) \mathrm{d} m} \\
& \times\left|y_{2}\left(t-\tau_{2}\right)-y_{2}^{*}\left(t-\tau_{2}\right)\right| \\
\leq & -\left\{\beta_{2}(t)\left[y_{2}(t)+y_{2}^{*}(t)\right]+c_{1}(t)\left[x_{2}(t)-x_{2}^{*}(t)\right]\right\} \\
& \times\left|y_{2}(t)-y_{2}^{*}(t)\right| \\
& +\alpha_{2}(t) e^{-\int_{t}^{t+\tau_{2}} \gamma_{2}(m) \mathrm{d} m}\left|y_{2}(t)-y_{2}^{*}(t)\right| .
\end{aligned}
$$

Let

$$
V(t)=V_{1}(t)+q V_{2}(t),
$$

where $q$ is a positive constant.

By calculating the upper right derivative of $V(t)$ along the positive $\omega$-periodic solution of model system (4) based on (93) and (95), it can be obtained as follows:

$$
\begin{gathered}
D^{+} V(t) \leq\left|x_{2}(t)-x_{2}^{*}(t)\right| \\
\times\left\{\alpha_{1}(t) e^{-\int_{t}^{t+\tau_{1}} \gamma_{1}(m) \mathrm{d} m}-\beta_{1}(t)\left[x_{2}(t)+x_{2}^{*}(t)\right]\right. \\
+\left[y_{2}(t)-y_{2}^{*}(t)\right]\left[c_{1}(t)+\rho(t)\right. \\
\times\left(\left(x_{2}(t)-x_{2}^{*}(t)\right) y_{2}(t)\right. \\
+\left(y_{2}(t)-y_{2}^{*}(t)\right) \\
\left.\left.\left.\times x_{2}^{*}(t)\right)\right]\right\} \\
+\left|y_{2}(t)-y_{2}^{*}(t)\right|\left\{q \alpha_{2}(t) e^{-\int_{t}^{t+\tau_{2}} \gamma_{2}(m) \mathrm{d} m}-q \beta_{2}(t)\right. \\
\times\left[y_{2}(t)+y_{2}^{*}(t)\right] \\
\left.-q c_{1}(t)\left[x_{2}(t)-x_{2}^{*}(t)\right]\right\} .
\end{gathered}
$$

According to Theorem 7, there exists a positive value $T>$ 0 , when $t \geq T$; we get that

$$
\begin{array}{ll}
m_{2}^{*}-\epsilon<x_{2}(t)<M_{2}^{*}+\epsilon, & m_{2}^{*}-\epsilon<x_{2}^{*}(t)<M_{2}^{*}+\epsilon, \\
m_{4}^{*}-\epsilon<y_{2}(t)<M_{4}^{*}+\epsilon, & m_{4}^{*}-\epsilon<y_{2}^{*}(t)<M_{4}^{*}+\epsilon
\end{array}
$$

hold for sufficiently small $\epsilon>0$.

Based on (98), when $t>T+\max \left\{\tau_{1}, \tau_{2}\right\}$, it is derived that

$$
\begin{aligned}
D^{+} V(t) \leq & -\left(G_{1}(t)-\epsilon\right)\left|x_{2}(t)-x_{2}^{*}(t)\right| \\
& -\left(G_{2}(t)-\epsilon\right)\left|y_{2}(t)-y_{2}^{*}(t)\right|,
\end{aligned}
$$

where $G_{1}(t)$ and $G_{2}(t)$ have been defined in Theorem 12 .

If $\liminf \inf _{t \rightarrow+\infty} G_{k}(t)>0$ for $k=1,2$, then there exist two constants $\delta_{1}>0$ and $\delta_{2}>0$ such that for $t \geq T^{*}:=$ $T+2 \max \left\{\tau_{1}, \tau_{2}\right\}$

$$
G_{1}(t) \geq \delta_{1}>0, \quad G_{2}(t) \geq \delta_{2}>0 .
$$

Consequently, for $t \geq T^{*}$, we have

$$
D^{+} V(t) \leq-\frac{\delta_{1}}{2}\left|x_{2}(t)-x_{2}^{*}(t)\right|-\frac{\delta_{2}}{2}\left|y_{2}(t)-y_{2}^{*}(t)\right| \text {. }
$$

By integrating both sides of (101) on the interval $\left[T^{*}, t\right]$, it can be obtained that, for $t \geq T^{*}$,

$$
\begin{aligned}
V(t) & +\frac{\delta_{1}}{2} \int_{T^{*}}^{t}\left|x_{2}(s)-x_{2}^{*}(s)\right| \mathrm{d} s \\
& +\frac{\delta_{2}}{2} \int_{T^{*}}^{t}\left|y_{2}(s)-y_{2}^{*}(s)\right| \mathrm{d} s \leq V\left(T^{*}\right) .
\end{aligned}
$$

Hence, $V(t)$ is bounded on the interval $\left[T^{*},+\infty\right)$ and

$$
\begin{aligned}
& \int_{T^{*}}^{t}\left|x_{2}(s)-x_{2}^{*}(s)\right| \mathrm{d} s<+\infty, \\
& \int_{T^{*}}^{t}\left|y_{2}(s)-y_{2}^{*}(s)\right| \mathrm{d} s<+\infty .
\end{aligned}
$$

According to Barbalat's Lemma [31], it can be concluded that

$$
\lim _{t \rightarrow \infty}\left|x_{2}(t)-x_{2}^{*}(t)\right|=0, \quad \lim _{t \rightarrow \infty}\left|y_{2}(t)-y_{2}^{*}(t)\right|=0 .
$$

It follows from (19) and (23) that

$$
\begin{aligned}
\left|x_{1}(t)-x_{1}^{*}(t)\right| & \leq \int_{t-\tau_{1}}^{t} \alpha_{1}(s) e^{\int_{t}^{s} \gamma_{1}(m) \mathrm{d} m}\left|x_{2}(s)-x_{2}^{*}(s)\right| \mathrm{d} s \\
& \leq \int_{t-\tau_{1}}^{t} \alpha_{1}^{M}\left|x_{2}(s)-x_{2}^{*}(s)\right| \mathrm{d} s, \\
\left|y_{1}(t)-y_{1}^{*}(t)\right| & \leq \int_{t-\tau_{2}}^{t} \alpha_{2}(s) e^{\int_{t}^{s} \gamma_{2}(m) \mathrm{d} m}\left|y_{2}(s)-y_{2}^{*}(s)\right| \mathrm{d} s \\
& \leq \int_{t-\tau_{2}}^{t} \alpha_{2}^{M}\left|y_{2}(s)-y_{2}^{*}(s)\right| \mathrm{d} s .
\end{aligned}
$$

Based on (104) and (105), it can be concluded that

$$
\lim _{t \rightarrow \infty}\left|x_{1}(t)-x_{1}^{*}(t)\right|=0, \quad \lim _{t \rightarrow \infty}\left|y_{1}(t)-y_{1}^{*}(t)\right|=0 .
$$

Therefore, it follows from (104) and (106) that model system (4) with initial conditions (5) and (6) has a unique positive $\omega$-periodic globally stable solution $\left(x_{1}^{*}(t)\right.$, $\left.x_{2}^{*}(t), y_{1}^{*}(t), y_{2}^{*}(t)\right)^{T}$.

3.4. Numerical Simulation. In this subsection, numerical simulations are carried out to substantiate the analytical findings obtained this paper. In order to facilitate the numerical simulations, $\omega$-periodic continuous functions introduced in model system (4) are selected as follows: $\alpha_{1}(t)=2.1+$ $\sin (t) / 10, \gamma_{1}(t)=0.2+\sin (t) / 200, \beta_{1}(t)=1+\sin (t) / 300$, $c_{1}(t)=0.2+\sin (t) / 300, \rho(t)=0.05+\sin (t) / 400, \alpha_{2}(t)=4.1+$ $\sin (t) / 18, \gamma_{2}(t)=0.1+\sin (t) / 580, \beta_{2}(t)=0.3+\sin (t) / 30$, and 

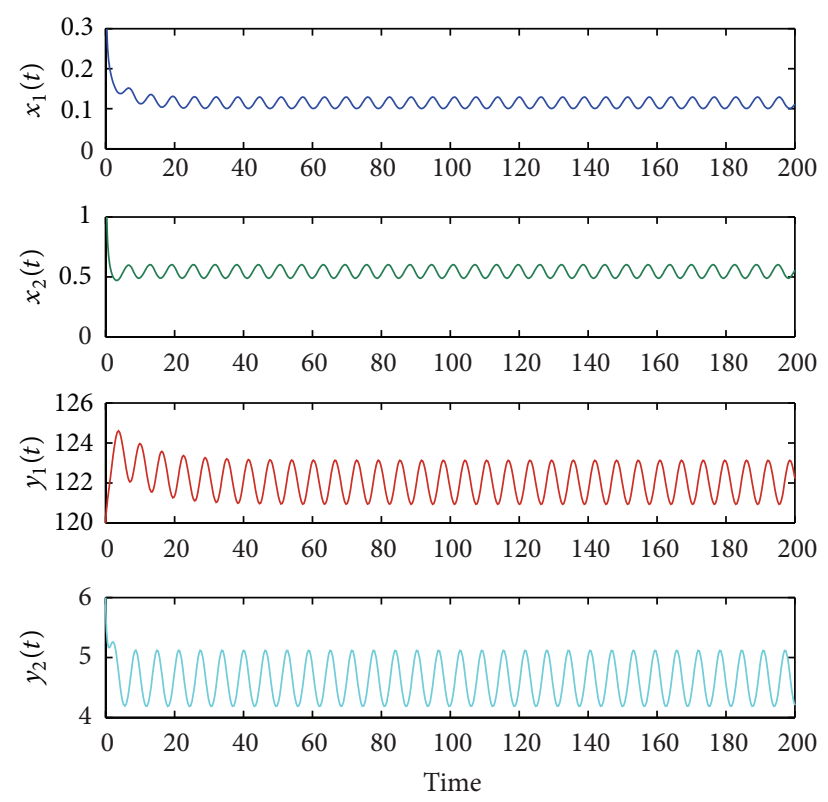

FIGURE 1: Dynamical responses of the unique positive $2 \pi$-periodic globally stable solution of model system (4).

$c_{2}(t)=0.15+\sin (t) / 270$. The maturation delay for nontoxic species and toxin liberating species is given as follows: $\tau_{1}=$ 0.1 and $\tau_{2}=0.2$, respectively. By using straightforward computation, it can be found that $\alpha_{1}^{L} \beta_{2}^{L} e^{-\gamma_{2}^{M} \tau_{1}}>\alpha_{2}^{M} c_{1}^{M} e^{-\gamma_{2}^{L} \tau_{2}}$, $\alpha_{2}^{L} \beta_{1}^{L} e^{-\gamma_{2}^{M} \tau_{2}}>\alpha_{1}^{M} c_{2}^{M} e^{-\gamma_{1}^{L} \tau_{1}}$; then model system (4) has at least one positive $\omega$-periodic solution based on Theorem 11 . Further computations show that $G_{1}(t) \geq 0.4281$ and $G_{2}(t) \geq 1.4006$. Consequently, it follows from Theorem 12 that model system (4) has a unique positive $2 \pi$-periodic globally stable solution $\left(x_{1}^{*}(t), x_{2}^{*}(t), y_{1}^{*}(t), y_{2}^{*}(t)\right)^{T}$, whose dynamical responses are plotted in Figure 1. Furthermore, the unique positive $2 \pi$-periodic globally stable solution $\left(x_{1}^{*}(t), x_{2}^{*}(t), y_{1}^{*}(t), y_{2}^{*}(t)\right)^{T}$ is plotted in the $x_{1}-x_{2}$ plane and $y_{1}-y_{2}$ plane, which can be found in Figures 2 and 3, respectively. It should be noted that two different initial solutions are included to show the attractivity of different solutions.

\section{Conclusion}

In this paper, a nonautonomous dynamical model is proposed to investigate population dynamics of competitive system with toxin liberating species and nontoxic species, where stage structure and maturation delay for two species are considered. It is well known that the effect of toxin ecological systems is an important issue from mathematical and experimental points of view. Generally speaking, it takes some time for a species to reach maturity to produce the toxicant, the toxin liberating mature individual produces a substance toxic to the nontoxic mature individuals only, and the inhibiting effect is zero in absence of either species. Furthermore, the species compete each other for the limited life resource within closed environment, but this competition only happens among the mature individuals and does not

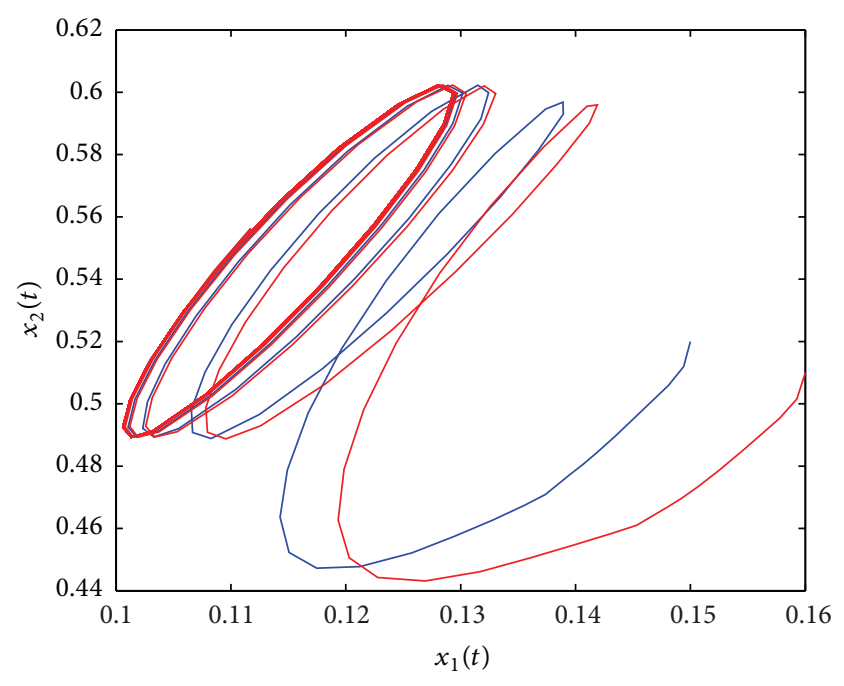

FIGURE 2: The unique positive $2 \pi$-periodic globally stable solution, which is plotted in the $x_{1}-x_{2}$ plane. Two different initial solutions are included to show the attractivity of different solutions, which are plotted in red and blue color, respectively.

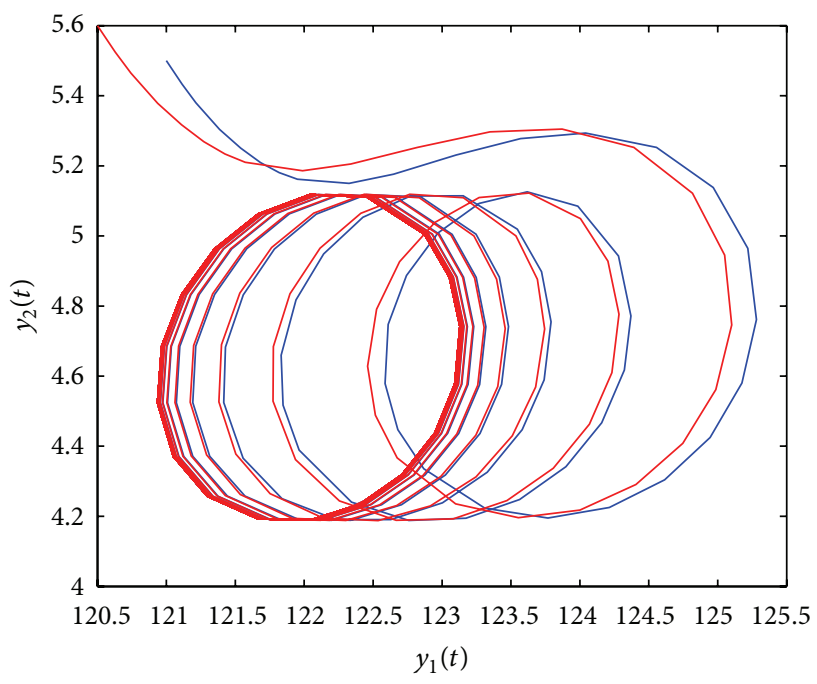

FIGURE 3: The unique positive $2 \pi$-periodic globally stable solution, which is plotted in the $y_{1}-y_{2}$ plane. Two different initial solutions are included to show the attractivity of different solutions, which are plotted in red and blue color, respectively.

involve the immature individuals. Consequently, it is necessary to investigate the dynamic effect of stage structure and toxic substances on population dynamics of two-species competitive system.

Qualitative analysis of the proposed model system is discussed in the third section of this paper. It follows from Theorems 1 and 2 that solutions of model system (4) with initial conditions are positive and ultimately bounded. By utilizing some comparison arguments, an iterative technique is proposed to discuss permanence of the species; model system (4) is persistent, which can be found in Theorem 7. 
Furthermore, existence of positive periodic solutions is considered in Theorem 11 based on continuation theorem of coincidence degree theory, which shows that model system (4) has at least one positive $\omega$-solution. By constructing an appropriate Lyapunov functional, sufficient conditions for global stability of the unique positive periodic solution are analyzed; that is, $\liminf _{t \rightarrow+\infty} G_{k}(t)>0, k=1,2$, which can be found in Theorem 12. Finally, numerical simulations are provided to show dynamical responses of the unique positive $2 \pi$-periodic globally stable solution, which are plotted in Figure 1. Furthermore, the unique positive $2 \pi$-periodic globally stable solution is plotted in the $x_{1}-x_{2}$ plane and $y_{1}-y_{2}$ plane, which can be found in Figures 2 and 3 , respectively. Since biological phenomenon associated with stage structure and toxin substances extensively exists within competitive system, theoretical results obtained in this paper are theoretically beneficial to discuss dynamic effect of maturation delay and toxic effect on population dynamics; it makes this work done in this paper has some positive and new features.

\section{Conflict of Interests}

All authors of this paper declare that there is no conflict of interests regarding the publication of this paper. They have no proprietary, financial, professional, or other personal interests of any nature or kind in any product, service, or company that could be construed as influencing the position presented in or the review of this paper.

\section{Acknowledgments}

The authors gratefully acknowledge anonymous reviewer and editor's comments. This work is supported by the National Natural Science Foundation of China, Grant nos. 61104003, 61273008, and 61104093, Research Foundation for Doctoral Program of Higher Education of Education Ministry, Grant no. 20110042120016, Hebei Province Natural Science Foundation, Grant no. F2011501023, Fundamental Research Funds for the Central Universities, Grant no. N120423009, and Research Foundation for Science and Technology Pillar Program of Northeastern University at Qinhuangdao, Grant no. XNK201301. This work is supported by State Key Laboratory of Integrated Automation of Process Industry, Northeastern University, Hong Kong Admission Scheme for Mainland Talents and Professionals, and Hong Kong Special Administrative Region.

\section{References}

[1] W. G. Aiello and H. I. Freedman, "A time-delay model of singlespecies growth with stage structure," Mathematical Biosciences, vol. 101, no. 2, pp. 139-153, 1990.

[2] G. Z. Zeng, L. S. Chen, L. H. Sun, and Y. Liu, "Permanence and the existence of the periodic solution of the non-autonomous two-species competitive model with stage structure," Advances in Complex Systems, vol. 7, no. 3-4, pp. 385-393, 2004.

[3] R. Xu, M. A. J. Chaplain, and F. A. Davidson, "Modelling and analysis of a competitive model with stage structure,"
Mathematical and Computer Modelling, vol. 41, no. 2-3, pp. 159175, 2005.

[4] D. Xu and X. Zhao, "Dynamics in a periodic competitive model with stage structure," Journal of Mathematical Analysis and Applications, vol. 311, no. 2, pp. 417-438, 2005.

[5] S. Ahmad and A. C. Lazer, "Average growth and total permanence in a competitive Lotka-Volterra system," Annali di Matematica Pura ed Applicata, vol. 185, pp. S47-S67, 2006.

[6] Z. Hou, "On permanence of all subsystems of competitive Lotka-Volterra systems with delays," Nonlinear Analysis: Real World Applications, vol. 11, no. 5, pp. 4285-4301, 2010.

[7] Z. G. Lin, "Time delayed parabolic system in a two-species competitive model with stage structure," Journal of Mathematical Analysis and Applications, vol. 315, no. 1, pp. 202-215, 2006.

[8] F. Chen, "Almost periodic solution of the non-autonomous two-species competitive model with stage structure," Applied Mathematics and Computation, vol.181, no. 1, pp. 685-693, 2006.

[9] Z. Liu, M. Fan, and L. Chen, "Globally asymptotic stability in two periodic delayed competitive systems," Applied Mathematics and Computation, vol. 197, no. 1, pp. 271-287, 2008.

[10] X. Xiong and Z. Zhang, "Periodic solutions of a discrete twospecies competitive model with stage structure," Mathematical and Computer Modelling, vol. 48, no. 3-4, pp. 333-343, 2008.

[11] M. Kouche, N. Tatar, and S. Liu, "Permanence and existence of a positive periodic solution to a periodic stage-structured system with infinite delay," Applied Mathematics and Computation, vol. 202, no. 2, pp. 620-638, 2008.

[12] B. D. Tian, Y. H. Qiu, and N. Chen, "Periodic and almost periodic solution for a non-autonomous epidemic predatorprey system with time-delay," Applied Mathematics and Computation, vol. 215, no. 2, pp. 779-790, 2009.

[13] H. Hu, Z. Teng, and H. Jiang, "On the permanence in nonautonomous Lotka-Volterra competitive system with puredelays and feedback controls," Nonlinear Analysis: Real World Applications, vol. 10, no. 3, pp. 1803-1815, 2009.

[14] F. Y. Wei, Y. R. Lin, L. L. Que, Y. Y. Chen, Y. P. Wu, and Y. F. Xue, "Periodic solution and global stability for a nonautonomous competitive Lotka-Volterra diffusion system," Applied Mathematics and Computation, vol. 216, no. 10, pp. 3097-3104, 2010.

[15] J. F. M. Al-Omari and S. K. Q. Al-Omari, "Global stability in a structured population competition model with distributed maturation delay and harvesting," Nonlinear Analysis. Real World Applications, vol. 12, no. 3, pp. 1485-1499, 2011.

[16] C. L. Shi, Z. Li, and F. D. Chen, "Extinction in a nonautonomous Lotka-Volterra competitive system with infinite delay and feedback controls," Nonlinear Analysis: Real World Applications, vol. 13, no. 5, pp. 2214-2226, 2012.

[17] Y. Li and Y. Ye, "Multiple positive almost periodic solutions to an impulsive non-autonomous Lotka-Volterra predator-prey system with harvesting terms," Communications in Nonlinear Science and Numerical Simulation, vol. 18, no. 11, pp. 3190-3201, 2013.

[18] J. D. Zhao, Z. C. Zhang, and J. Ju, "Necessary and sufficient conditions for permanence and extinction in a three dimensional competitive Lotka-Volterra system," Applied Mathematics and Computation, vol. 230, pp. 587-596, 2014.

[19] H. Zhang, Y. Li, B. Jing, and W. Zhao, "Global stability of almost periodic solution of multispecies mutualism system with time delays and impulsive effects," Applied Mathematics and Computation, vol. 232, no. 1, pp. 1138-1150, 2014. 
[20] H. R. Thieme, Mathematics in Population Biology, Princeton University Press, Princeton, NJ, USA, 2003.

[21] G. P. Samanta, "A two-species competitive system under the influence of toxic substances," Applied Mathematics and Computation, vol. 216, no. 1, pp. 291-299, 2010.

[22] J. Maynard Smith, Models in Ecology, Cambridge University Press, Cambridge, UK, 1974.

[23] M. Bandyopadhyay, "Dynamical analysis of a allelopathic phytoplankton model," Journal of Biological Systems, vol. 14, no. 2, pp. 205-217, 2006.

[24] Z. E. Ma, G. R. Cui, and W. D. Wang, "Persistence and extinction of a population in a polluted environment," Mathematical Biosciences, vol. 101, no. 1, pp. 75-97, 1990.

[25] W. D. Wang and Z. E. Ma, "Permanence of populations in a polluted environment," Mathematical Biosciences, vol. 122, no. 2, pp. 235-248, 1994.

[26] J. Zhen and Z. E. Ma, "Periodic solutions for delay differential equations model of plankton allelopathy," Computers \& Mathematics with Applications, vol. 44, no. 3-4, pp. 491-500, 2002.

[27] F. Wang and Z. Ma, "Persistence and periodic orbits for an SIS model in a polluted environment," Computers \& Mathematics with Applications, vol. 47, no. 4-5, pp. 779-792, 2004.

[28] Z. Li and F. D. Chen, "Extinction in periodic competitive stage-structured Lotka-Volterra model with the effects of toxic substances," Journal of Computational and Applied Mathematics, vol. 231, no. 1, pp. 143-153, 2009.

[29] X. Y. Song and L. S. Chen, "Optimal harvesting and stability for a two-species competitive system with stage structure," Mathematical Biosciences, vol. 170, no. 2, pp. 173-186, 2001.

[30] W. Wang, G. Mulone, F. Salemi, and V. Salone, "Permanence and stability of a stage-structured predator-prey model," Journal of Mathematical Analysis and Applications, vol. 262, no. 2, pp. 499528, 2001.

[31] R. E. Gaines and J. L. Mawhin, Coincidence Degree and Nonlinear Differential Equations, Springer, Berlin, Germany, 1977. 


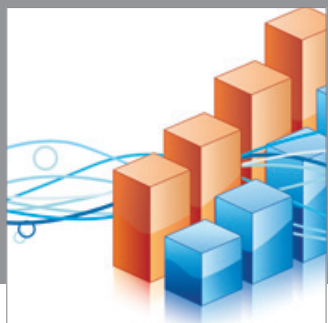

Advances in

Operations Research

mansans

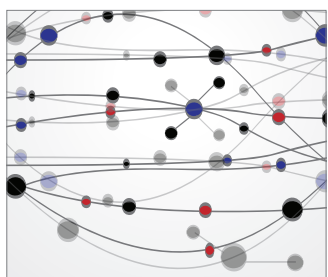

The Scientific World Journal
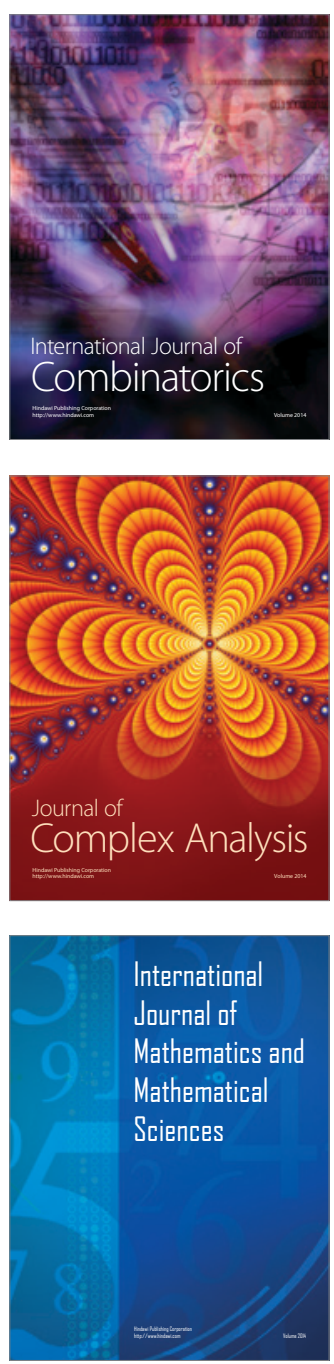
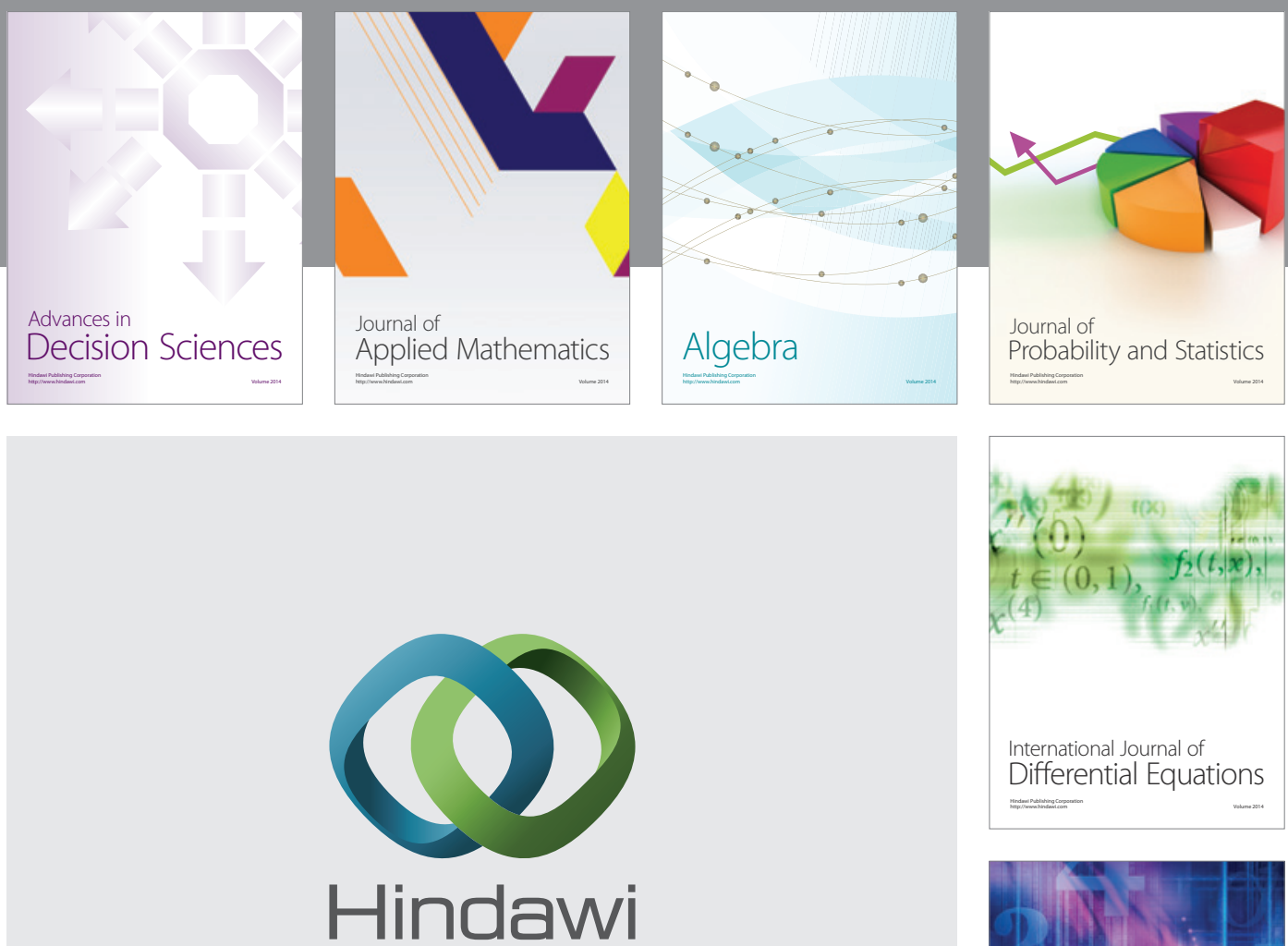

Submit your manuscripts at http://www.hindawi.com
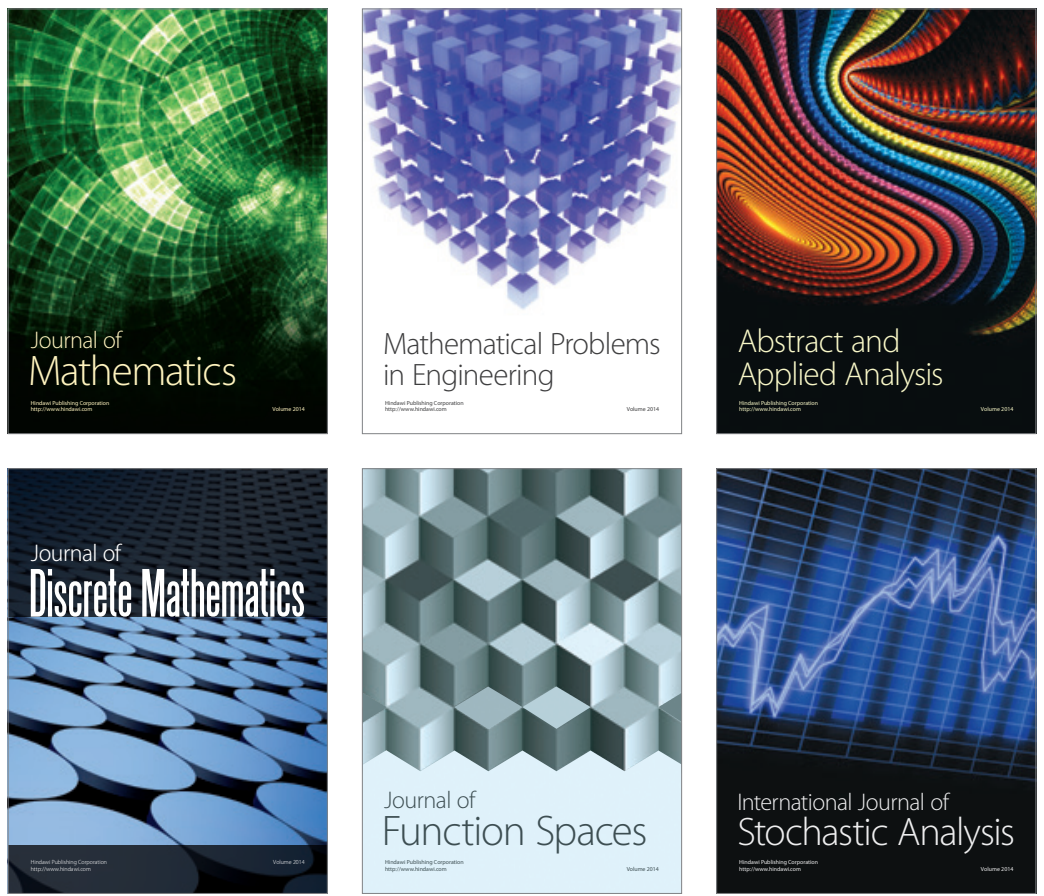

Journal of

Function Spaces

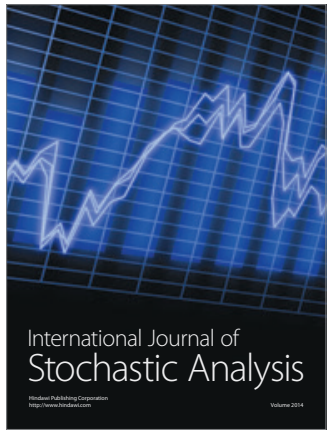

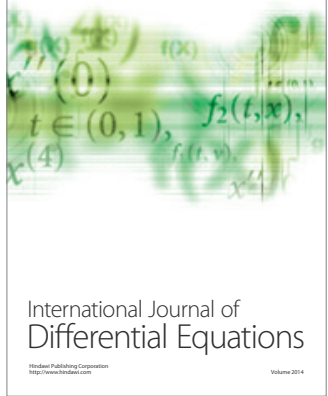
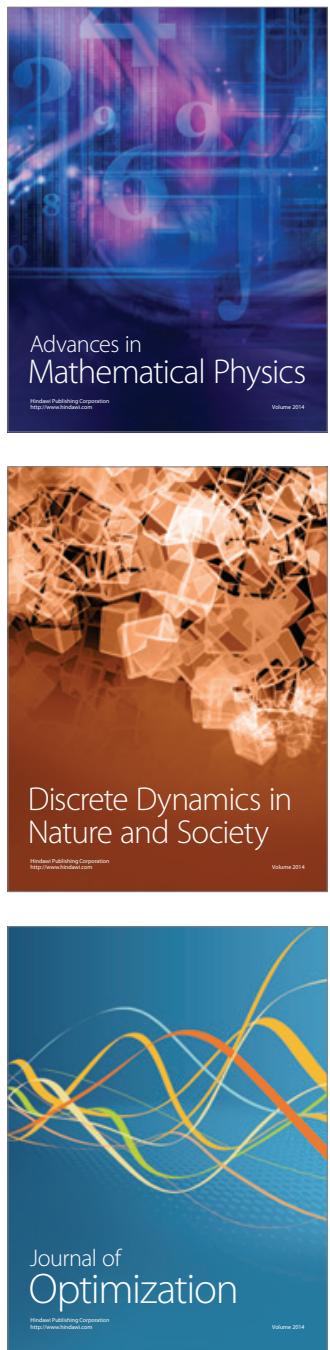